\title{
INFLUENCE OF FRACTURE STABILITY ON STAPHYLOCOCCUS EPIDERMIDIS AND STAPHYLOCOCCUS AUREUS INFECTION IN A MURINE FEMORAL FRACTURE MODEL
}

\author{
M. Sabaté Brescó1,2, L. O’Mahony², S. Zeiter ${ }^{1}$, K. Kluge ${ }^{1}$, M. Ziegler², C. Berset ${ }^{3}$, D. Nehrbass ${ }^{1}$, \\ R. G. Richards ${ }^{1}$ and T. F. Moriarty ${ }^{1, *}$ \\ ${ }^{1}$ AO Research Institute Davos, Davos, Switzerland \\ ${ }^{2}$ Swiss Institute of Allergy and Asthma Research, Davos, Switzerland \\ ${ }^{3}$ AO Foundation, Davos, Switzerland
}

\begin{abstract}
Fracture-related infection (FRI) is a major complication in surgically fixed fractures. Instability of the fracture after fixation is considered a risk factor for infection; however, few experimental data are available confirming this belief. To study whether stable fractures led to higher infection clearance, mouse femoral osteotomies were fixed with either stable or unstable fixation and the surgical site was contaminated with either Staphylococcus epidermidis (S. epidermidis) or Staphylococcus aureus (S. aureus) clinical isolates. Infection progression was assessed at different time points by quantitative bacteriology, total cell counts in spleen and lymph node and histological analysis. Operated, non-inoculated mice were used as controls. Two inbred mouse strains (C57BL/6 and BALB/c) were included in the study to determine the influence of different host background in the outcome.

Stable fixation allowed a higher proportion of C57BL/6 mice to clear S. epidermidis inoculation in comparison to unstable fixation. No difference associated with fixation type was observed for BALB/c mice. Inoculation with $S$. aureus resulted in a more severe infection for both stable and unstable fractures in both mouse strains; however, significant osteolysis around the screws rendered the stable group functionally unstable.

Our results suggested that fracture stability could have an influence on S. epidermidis infection, although host factors also played a role. No differences were observed when using $S$. aureus, due to a more severe infection, leading to osteolysis and loss of stability in both groups. Further studies are required in order to address the biological features underlying the differences observed.
\end{abstract}

Keywords: Bone infection, S. epidermidis, S. aureus, mouse model, trauma, fracture stability, stable fixation, unstable fixation.

*Address for correspondence: T.F. Moriarty, AO Research Institute Davos, AO Foundation, Clavadelerstrasse 8, Davos Platz, 7270, Switzerland.

Phone: +41814142397 Email: fintan.moriarty@aofoundation.org

\section{Introduction}

Fracture-related infection (FRI) remains one of the most significant complications in the surgical treatment of fractured bones. FRI is often associated with prolonged antibiotic treatment, repeated surgical debridement, implant removal, high healthcare costs and poor functional outcome for affected patients (Metsemakers et al., 2016; Willey and Karam, 2016). The prevalence of FRI ranges from $0.5-3 \%$ for closed fractures, but can increase up to $30 \%$ in open fractures (Metsemakers et al., 2016; Uckay et al., 2013). Staphylococcus aureus (S. aureus) and the coagulase-negative staphylococci (CoNS), in particular Staphylococcus epidermidis (S. epidermidis), are the most prevalent species in FRI, accounting together for 50-75\% of all cases (Montanaro et al., 2011; Trampuz and Zimmerli, 2006).
The risk of developing an infection associated with any implanted device is increased by the presence of the device itself (Elek and Conen, 1957; James and Macleod, 1961), which acts as a foreign body, providing a surface for adhesion and biofilm formation to any microorganism entering the wound. Other risk factors for implant-associated infections include patient characteristics such as advanced age, smoking or co-morbidities such as diabetes or obesity (Jeffcoach et al., 2014; Moucha et al., 2011; Willey and Karam, 2016). The biomechanical properties of the fixation are additional specific factors in surgicallyfixed fractures. It is widely known that healing of fractures is influenced by biomechanics (Augat et al., 2005; Glatt et al., 2016; Lacroix and Prendergast, 2002): when fracture fixation provides absolute stability, the fragments are compressed together with little strain under loading, leading to primary bone healing; in 
contrast, when the fixation is less rigid, displacement may occur under load and the fracture tends to heal due to secondary bone healing, with callus formation (Claes et al., 2012; Grongroft et al., 2009). The stability of the fixation is also considered to be a critical factor in FRI (Mouzopoulos et al., 2011; Rittman and Perren, 1974). A few preclinical experimental studies (Friedrich and Klaue, 1977; Merritt and Dowd, 1987; Rittman and Perren, 1974; Worlock et al., 1994) provide supportive evidence for the association between stable fixation and reduced infection risk. However, there is poor standardisation of the stability of the fractures within these models and no studies to date have investigated this phenomenon for $S$. epidermidis FRI. S. aureus FRI has been intensively studied in both clinical and basic science, with numerous preclinical in vivo models available (Arens et al., 2015; Lindsey et al., 2010; Robinson et al., 2011; Rochford et al., 2016; Windolf et al., 2013; Worlock et al., 1988). However, the literature describing $S$. epidermidis FRI is comparatively scarce and fewer in vivo preclinical models are available (Lovati et al., 2017).

The aim of the present study was, therefore, to study FRI progression under different mechanical environments (stable and unstable fixation). In order to provide the controlled mechanical environment necessary, an established mouse implant system (MouseFix ${ }^{\mathrm{TM}}$ ) was used, which provides two types of implants, rigid and flexible (Grongroft et al., 2009; Matthys and Perren, 2009; Steck et al., 2011). Previous studies using these implants show that under rigid fixation, bone healing occurs through a mixture of intramembranous and some endochondral ossification, while flexible fixation results in endochondral ossification and the formation of a large stabilising callus at day 14 (Grongroft et al., 2009; Steck et al., 2011). Ex vivo mechanical testing confirms that the flexible plate provides only $1 / 4$ of the stiffness of the rigid plate in a vertical plane (Grongroft et al., 2009). Two well-characterised and widely used inbred mouse strains were used in this study. C57BL/6 mice were selected as they have been used in previous studies with MouseFix ${ }^{\mathrm{TM}}$ implants. Since this strain is Thelper (Th) 1-skewed, a Th2-skewed strain (BALB/c) was included to determine if the observations were consistent across different host backgrounds.

\section{Materials and methods}

\section{Animals}

The study was approved by the ethical committee of the canton of Grisons in Switzerland (TVB 11/2013) and was carried out in a research facility accredited by the Association for Assessment and Accreditation for Laboratory Animal Care (AAALAC) International. Skeletally mature [20-28 weeks old, average weight \pm standard deviation (SD): $24.34 \pm 2.12 \mathrm{~g}$ ], female, specific pathogen free (SPF) C57BL/6 and BALB/C mice (Charles River, Germany) were used in this study. All animals were allowed to acclimatise to experimental conditions for at least two weeks prior to the start of the study. Mice were randomly housed in individually ventilated cages (XJ, Allentown, PA, USA) under a $12 \mathrm{~h}$ dark/light cycle in groups of three to six. Cages were changed weekly and mice were re-housed in the same groups post-surgery. Cages were randomly assigned to the different groups and all animals within the same cage were assigned to the same treatment group. Animals were fed with a standard diet (3436, Provimi Kliba, Kaiseraugst, Switzerland) and had free access to sterile water ad libitum. Any animal excluded from the experiment due to fracture or implant failure was replaced unless stated otherwise.

\section{Study design}

The study was performed comparing rigid to flexible fixation, in groups of mice, according to the scheme outlined in Fig. 1. The first series of experiments compared rigid fixation and flexible fixation in C57BL/6 (Fig. 1a) and BALB/c (Fig. 1b) mice, under aseptic (non-inoculated) conditions or inoculated with S. epidermidis (Fig. 1a,b). The primary outcome was infection rate and quantitative measurement of bacterial burden [colony forming units (CFU)], with additional animals used to obtain histological samples. The second series of experiments involved inoculation with $S$. aureus, which was performed at the standard dose in C57BL/6 mice (Fig. 1c), but that series was discontinued due to rapid onset of osteolysis leading to unstable fractures in both groups. Therefore, the experiment was repeated in C57BL/6 and BALB/C mice at lower doses (Fig. 1d). Age-matched C57BL/6 and BALB/c mice $(n=6-8)$, without any surgical intervention, were euthanised in the different experiments as controls to obtain baseline levels of cell counts in different organs and to assess inter-experimental variability.

\section{Bacteria and inoculum preparation}

The two tested microorganisms were S. epidermidis Epi 103.1 and S. aureus JAR06.01.31, both of which were clinical isolates. S. epidermidis Epi 103.1 was obtained from a patient with chronic implant-related bone infection (Trauma Centre BGU, Murnau, Germany), has a weak in vitro biofilm formation capacity, according to the scoring system of Stepanovic et al. (2000), and is resistant to benzylpenicillin and fusidic acid. S. aureus JAR06.01.31, was originally cultured from an infected hip prosthesis (University Hospital Basel, Basel, Switzerland) (Campoccia et al., 2008; Moriarty et al., 2009), belongs to sequence type (ST) 15 , displays weak biofilm formation capacity and is resistant only to benzylpenicillin. Both strains are deposited in the Culture Collection of Switzerland (CCOS): S. epidermidis Epi 103.1 (CCOS 1152) and S. aureus JAR06.01.31 (CCOS 890).

For inocula preparation, a single colony was resuspended in $40 \mathrm{~mL}$ of tryptone soy broth (TSB) (Oxoid, Postfach, Germany) and cultured overnight 
at $37^{\circ} \mathrm{C}$ under a $100 \mathrm{rpm}$ agitation. On the day of the surgery, the bacteria were sub-cultured into prewarmed TSB and incubated at $37^{\circ} \mathrm{C}$ under a $100 \mathrm{rpm}$ agitation for at least $2 \mathrm{~h}$ to obtain a log-phase culture. When a pre-defined optical density, corresponding to $4.6 \times 10^{7} \mathrm{CFU} / \mathrm{mL}$, was reached, the culture was centrifuged at $8,500 \times g$ for $5 \mathrm{~min}$ and resuspended in the same volume of phosphate buffered saline (PBS) (Sigma-Aldrich, St. Luis, MO, USA). Finally, the suspension was further diluted, as required, in PBS to prepare the inoculum, which was used within $1 \mathrm{~h}$. The target inoculum was set to $1 \times 10^{4} \mathrm{CFU}$ for the standard $S$. epidermidis and $S$. aureus dose, and $1 \times 10^{3}$ for the low $S$. aureus dose.

\section{Implants and surgery}

Commercially pure titanium (CpTi) 4-hole rigid and CpTi 4-hole flexible MouseFix ${ }^{\mathrm{TM}}$ implants (Fig. 2) and titanium aluminium niobium alloy [Ti6Al4Nb (TAN)] screws were used in this study (RISystem, Davos, Switzerland). The side bars of the flexible implant are composed of Nitinol. Prior to use, all implants were cleaned and sterilised as previously described (Rochford et al., 2016).

Surgery was performed as previously described (Rochford et al., 2016). Briefly, under general anaesthesia with isofluorane (2-3\% isoflurane in $100 \% \mathrm{O}_{2}, 1 \mathrm{~L} / \mathrm{min}$ ) (Isofluran Baxter ${ }^{\circledR}$, Baxter, Opfikon, Switzerland), the animal was placed in prone position and a skin incision was made from tail base to left stifle. The subcutaneous fascia lata was cut and the tissue plane between the quadriceps and the biceps femoris muscle was bluntly dissected. A Teflon ${ }^{\mathrm{TM}}$ foil was placed around the femur to protect the soft tissue from contamination. Then, the implant was fixed to the bone using four self-tapping, angular stable screws. Screw holes were predrilled with a $0.31 \mathrm{~mm}$ drill bit (RISystem). Once the implant was in place, a $0.44 \mathrm{~mm}$ defect osteotomy was performed using a jig and a Gigly wire. In the inoculated groups, $2.5 \mu \mathrm{L}$ of bacteria suspension were injected in the osteotomy site. Next, the foil was removed and the fascia lata and the skin were closed with continuous sutures (5-0 Vicryl rapide, Ethicon, Dilbeek, Belgium). Following surgery, a lateral radiograph of the femur was taken to confirm proper positioning of the implant.

Analgesia was provided in the form of subcutaneous injections of buprenorphine $(0.1 \mathrm{mg} / \mathrm{kg}$, Bupaq $^{\circledR}$, Streuli Pharma AG, Uznach, Switzerland), pre-operatively and post-operatively every $6-8 \mathrm{~h}$ for $16 \mathrm{~h}$. Additionally, a palatable form of acetaminophen ( $2 \mathrm{mg} / \mathrm{mL}$, as Dafalgan ${ }^{\circledR}$ Syrup for Children, BristolMyers Squibb SA, Cham, Switzerland) was added to the drinking water for $5 \mathrm{~d}$. Animals were monitored twice daily for $5 \mathrm{~d}$ post-operatively and subsequently once a day, using a detailed numerical scoring system, which took into account general behaviour, external appearance, wound status and animal movement (scale $0-18$, Table 1). Weights and radiographs of the operated limb were taken weekly.
Table 1. Self-designed animal scoring system.

\begin{tabular}{|l|l|}
\hline Parameter & Scores \\
\hline Behaviour/Overall impression & $0 / 2 / 4$ \\
\hline Breathing & $0 / 2 / 3$ \\
\hline Fur/Eyes/Skin & $0 / 1 / 3$ \\
\hline Movement & $0 / 1 / 2 / 3$ \\
\hline Faeces/Urine & $0 / 2$ \\
\hline
\end{tabular}

\section{Gait analysis}

Gait analysis was performed in a subset of noninoculated animals in order to compare weight bearing among animals receiving different implant types. Mice ( $n=5$ per group) were made run through the Catwalk XT system (Noldus Information Technology, Wageningen, Netherlands) (Hamers et al., 2001) for the detection of paw footprints (camera gain was set to 20 and the detection threshold to 0.1 ). Three runs with a speed variance lower than $60 \%$ were recorded per animal pre-operatively and $6,9,12,24,48$ and $72 \mathrm{~h}$ after surgery. Footprint area $\left(\mathrm{cm}^{2}\right)$ and light intensity (arbitrary units) of detected paw footprints were the selected outcome measures.

\section{Euthanasia and post-mortem sample processing} At each scheduled time point, the animals were euthanised by cervical dislocation after inducing general anaesthesia in an induction box (5\% isoflurane in $100 \% \mathrm{O}_{2}, 1 \mathrm{~L} / \mathrm{min}$ ). A lateral radiograph of the left femur with implant in place was acquired post-mortem. The operated femur, the implant and the surrounding soft tissue were harvested separately in sterile containers containing cold PBS. The soft tissue and the bone were mechanically homogenised (Omni Tissue Homogenizer and Hard Tissue Homogenizing tips, Omni International, Kennesaw, GA, USA). The number of bacteria associated with the implant was determined by sonicating implant and screws at $40 \mathrm{kHz}$ for $3 \mathrm{~min}$ in PBS (Bandelin Sonorex, Berlin, Germany) and vortexing for $10 \mathrm{~s}$. Viable bacterial count was performed by plating serial dilutions of each sample on $5 \%$ horse blood agar (BA) (Oxoid). All BA plates were incubated at $37^{\circ} \mathrm{C}$ and bacteria count was performed 24 and $48 \mathrm{~h}$ later. Mice were considered as infected when at least one sample (bone, soft tissue or implant) was culture-positive. Bacteria recovered from each animal were confirmed to be S. epidermidis Epi 103.1 by Random Amplification of Polymorphic DNA (RAPD) test (Versalovic et al., 1991) or to be $S$. aureus by latex agglutination assay (Staphaurex, Thermo Fischer Scientific, Reinach, Switzerland). Spleen and popliteal lymph node were harvested in cold PBS and processed to obtain total cell counts in each organ as a measure of leukocyte infiltration, as previously described (Rochford et al., 2016). Animals with osteosynthesis or implant failure were excluded from final analysis and replaced when considered necessary (see Fig. 1 legend). 
Histology and scanning electron microscopy

Samples for histology (Fig. 1) were largely processed as described previously (Rochford et al., 2016). Immediately after euthanasia, the entire operated femur and surrounding musculature underwent fixation with $70 \%(\mathrm{v} / \mathrm{v})$ methanol for polymethylmethacrylate (PMMA) or paraffin embedding. PMMA samples were dehydrated through an ascending series of ethanol, transferred into xylene and finally infiltrated with and embedded in PMMA (Grongroft et al., 2009). The polymerised blocks were cut using an annular blade saw (Leica SP1600 saw microtome, Leica AG, Wetzlar, Germany). Two sections were glued onto opaque plexiglass slides using cyanoacrylate, ground, polished down to approximately $100 \mu \mathrm{m}$ using a microgrinding system (Exact ${ }^{\circledR}$, Frankfurt am Main, Germany) and stained with Giemsa/eosin (GE) staining. GE sections were blindly analysed by a certified pathologist.
For paraffin embedding, the implant was removed and samples were decalcified in a $12.5 \%$ EDTA, $1.25 \% \mathrm{NaOH}$ solution. Finally, samples were cut longitudinally and both halves were embedded together in paraffin wax (McCormick, St. Luis, MO, USA). Paraffin blocks were cut using a HM 355S microtome (Microm, Thermo Fisher Scientific, MA, USA) and sections were collected on Superfrost Plus slides (Thermo Fisher Scientific, USA). Slides were stained with Brown and Brenn (BB) for bacteria staining. The removed implants and selected paraffin sections (after deparaffinisation) were analysed by scanning electron microscopy (SEM) for observation of any bacteria. Afterwards, the samples were dehydrated in an ascending series of ethanol (50$100 \%, 2 \times 5 \mathrm{~min}$ ) and implants and sections were left to air-dry overnight in a sterile flow hood. Samples were sputter-coated with $10 \mathrm{~nm}$ gold palladium using a BAL-TEC MED 020 (BAL-TEC AG, Balzers,

$\mathbf{a}$

\begin{tabular}{|l|c|c|c|c|c|c|}
\hline & 3 & $\mathbf{7}$ & \multicolumn{2}{c}{$\mathbf{1 4}$} & \multicolumn{2}{c}{$\mathbf{3 0}$} \\
\hline Rigid non-inoculated & 6 & $>6$ & CFU counts & Histology & CFU counts & Histology \\
\hline Flexible non-inoculated & 6 & $>6$ & $>6$ & 6 & $>6$ & 4 \\
\hline Rigid SE inoculated & 6 & 6 & $>6$ & 6 & $>6$ & 3 \\
\hline Flexible SE inoculated & $>6$ & 5 & $>6$ & 6 & 6 & 4 \\
\hline
\end{tabular}

b

\begin{tabular}{l}
\hline \multicolumn{7}{c}{ Sx } \\
\hline
\end{tabular}

c

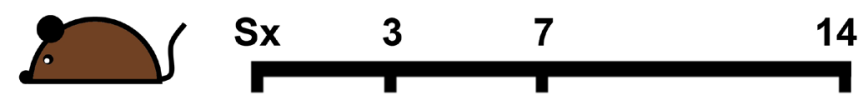

\begin{tabular}{|l|c|c|c|}
\multicolumn{1}{c}{ CFU counts } & CFU counts & CFU counts \\
\hline Rigid SA inoculated & 6 & $>6$ & $>6$ \\
\hline Flexible SA inoculated & $>6$ & $>6$ & $>6$ \\
\hline
\end{tabular}

d

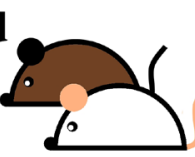

Sx

\begin{tabular}{|c|c|c|}
\hline & & CFU counts \\
\hline \multirow{2}{*}{$\begin{array}{l}\text { Rigid SA low } \\
\text { inoculated }\end{array}$} & C57BL/6 & $>6$ \\
\hline & $\mathrm{BALB} / \mathrm{c}$ & 5 \\
\hline \multirow{2}{*}{$\begin{array}{l}\text { Flexible SA low } \\
\text { inoculated }\end{array}$} & C57BL/6 & 6 \\
\hline & BALB/c & $>6$ \\
\hline
\end{tabular}

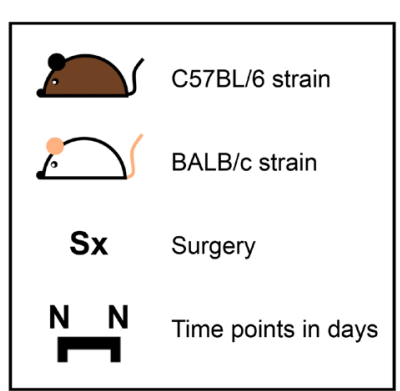

Fig. 1. Graphic scheme of animals per group in the different studies, with C57BL/6 represented as a brown mouse and BALB/c as a white mouse. Surgery $(\mathrm{Sx})$ and time points in day are indicated on each axis. SE: S. epidermidis, SA: S. aureus. N.B. day 3 was not performed in BALB/c as no clearance was observed either at day 7 or 14 , and, thus, it was not deemed necessary to evaluate impact of fixation on clearance at that time point. For the same reason, it was not deemed necessary to replace animals in some groups at day 7 and 14 (marked with an asterisk). For S. aureus low dose, only one time point was performed. Again, as no differences were observed in CFU counts and clearance, earlier time points were not studied. 
Liechtenstein). Representative SEM micrographs of the surfaces were acquired using a Hitachi FESEM

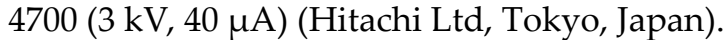

\section{Statistical analysis}

Statistical analysis was performed with GraphPad Prism6 (GraphPad, La Jolla, CA, USA). Fisher exact test was used for proportions of infected animals and Mann-Whitney test for quantitative CFU data. Weight change and cell counts were compared over time among groups with 2-way ANOVA with Sidak or Dunnett post hoc tests. Differences were considered statistically significant with a $p$-value $<0.05$.

\section{Results}

\section{Animal monitoring}

In total, 255 C57BL/6 mice and 135 BALB/c mice were operated on throughout the entire study. 38 (9.78\%) of the osteosyntheses/implants failed, resulting in early euthanasia. Additionally, 10 animals were excluded $(2.57 \%)$ due to post-operative complications not related to the fracture model (lameness, secondary infection or exitus) and 10 animals (2.57\%) were excluded due to intra-operative complications (such as implant misplacement, implant failure or anaesthetic fatality). These animals were excluded from analysis and replaced as deemed necessary (Fig. 1). No differences were observed between mouse strains or implant type in terms of susceptibility to failure. S. aureus-inoculated animals presented a higher rate of exclusion (17.14\%) compared to $S$. epidermidis-inoculated animals (10.15\%), although the difference was not statistically significant.

In general, there was no consistent difference for either C57BL/6 or BALB/C mice with regard to weight change among groups (rigid vs. flexible and non-inoculated vs. S. epidermidis-inoculated). In S. aureus-inoculated mice, no statistically significant differences were observed in weight loss between rigid and flexible groups. The greatest effect was seen in S. aureus-inoculated C57BL/6 and BALB/c mice, which lost more weight than their $S$. epidermidisinoculated and non-inoculated counterparts $(p<0.05$, Fig. 3). The clinical score did not show significant differences among groups.

\section{Gait analysis}

Gait analysis revealed that, during the first 9-12 $\mathrm{h}$ post-operative, there was an observable difference in the footprint area of the operated limb between mice fixed with rigid and flexible implants (Fig. 4). The footprint area returned to baseline level after this time. Of note, there was no significant difference between the rigid and flexible implant groups, although, for mice with a flexible device, the decrease was significant and returned to baseline level with a slight delay compared to rigidly fixed equivalents. There was a trend for increased values for the contralateral limb for the first $24 \mathrm{~h}$, remaining high up to $72 \mathrm{~h}$ post-operatively, which most likely was to compensate for the effect on the operated limb.

\section{Bacteriology}

Quantitative bacteriological analysis of the inocula prepared for the surgeries confirmed that they were within the target range. For S. epidermidis, the average inoculum was $1.35 \times 10^{4} \mathrm{CFU}\left(\mathrm{SD}: 5.69 \times 10^{3}\right.$ ) in C57BL/6 and $1.95 \times 10^{4} \mathrm{CFU}\left(\mathrm{SD}: 5.02 \times 10^{3}\right) \mathrm{in} \mathrm{BALB} / \mathrm{c}$. For $S$. aureus, the inoculum was $1.13 \times 10^{4} \mathrm{CFU}$ (SD: $\left.4.28 \times 10^{3}\right)$ for the high dose study and $1.98 \times 10^{3} \mathrm{CFU}$ $\left(\mathrm{SD}: 3.83 \times 10^{2}\right)$ for the low dose study. No significant differences were observed between the inocula administered to the rigid and flexible implant groups.

Post-mortem, mice were confirmed as infected or not-infected based on quantitative bacteriological evaluation of bone, soft tissue and implant from the operated limb. The results of the bacteriological assessment for C57BL/6 mice receiving S. epidermidis

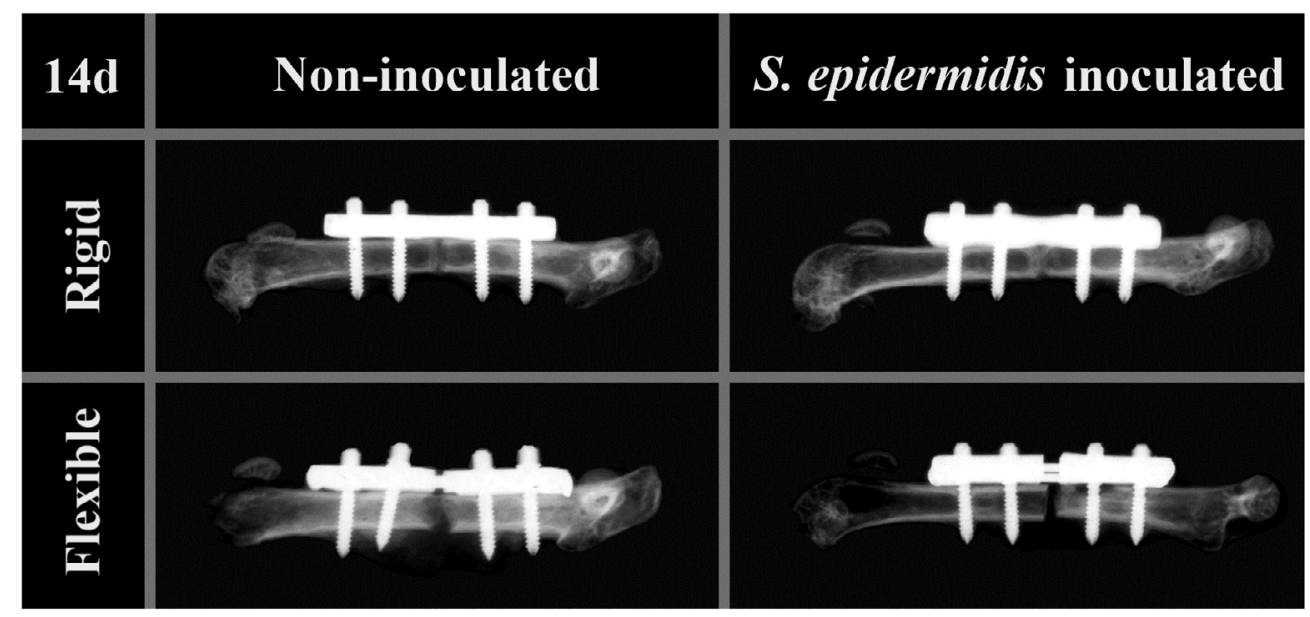

Fig. 2. Post-mortem contact radiographs of rigid and flexible plates in non-inoculated (left) and S. epidermidis inoculated (right) C57BL/6 mice at day 14 post-operatively. The specimens (operated femur with implant in place with surrounding soft tissues) were fixed in $70 \%$ ethanol previous to radiograph acquisition. N.B. osteotomy started to show closure in animals with rigid implant fixation, while no such closure was observed in animals with flexible fixation. 


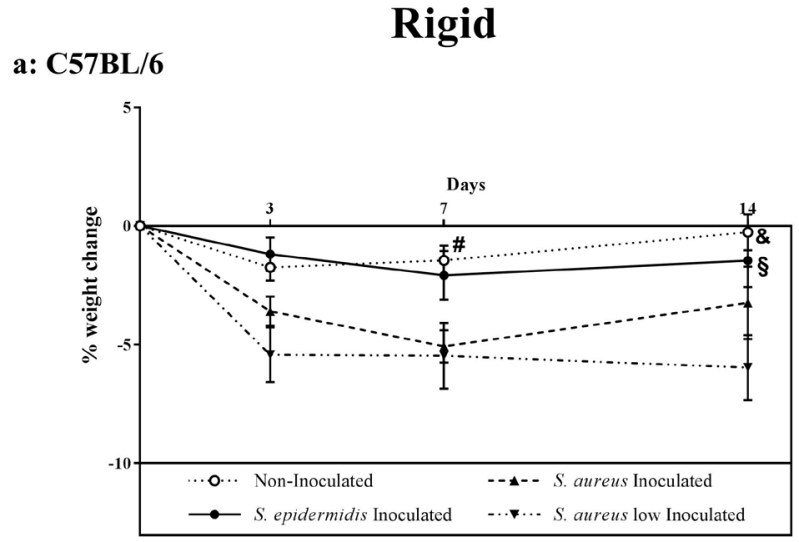

c: $B A L B / c$

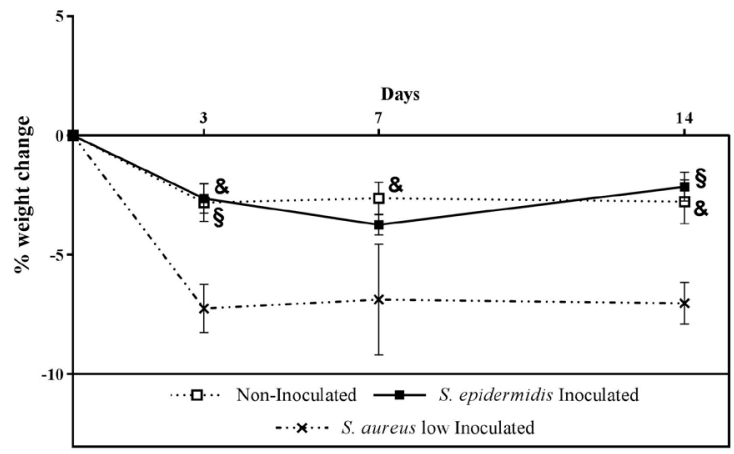

b: C57BL/6

Flexible

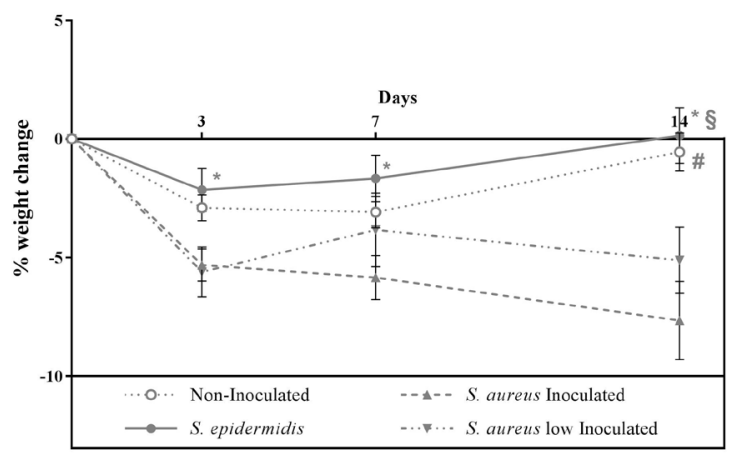

d: BALB/c

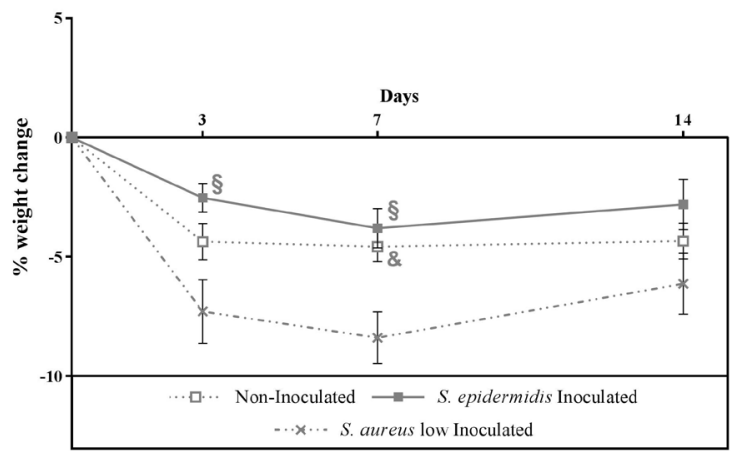

Fig. 3. Weight change of S. epidermidis- and S. aureus-infected $(\mathbf{a}, \mathbf{b})$ C57BL/6 and $(\mathbf{c}, \mathbf{d})$ BALB/c mice. Percentage of weight change (compared to post-operation weight) in (a) C57BL/6 mice with a rigid implant, (b) C57BL/6 mice with a flexible implant, (c) BALB/c mice with a rigid implant and (d) BALB/c mice with a flexible implant. Mean and SEM $(n=10-43)$. 2-way ANOVA with Tukey post hoc test; $\# / * / \& / \S=p<0.05$. Statistically significant differences are marked as follows: \# for differences between non-inoculated and S. aureus-inoculated, ${ }^{*}$ for differences between S. epidermidis-inoculated and S. aureus-inoculated, \& for differences between non-inoculated and $S$. aureus low-dose-inoculated and $\S$ for differences between $S$. epidermidis-inoculated and S. aureus low-dose-inoculated.

a

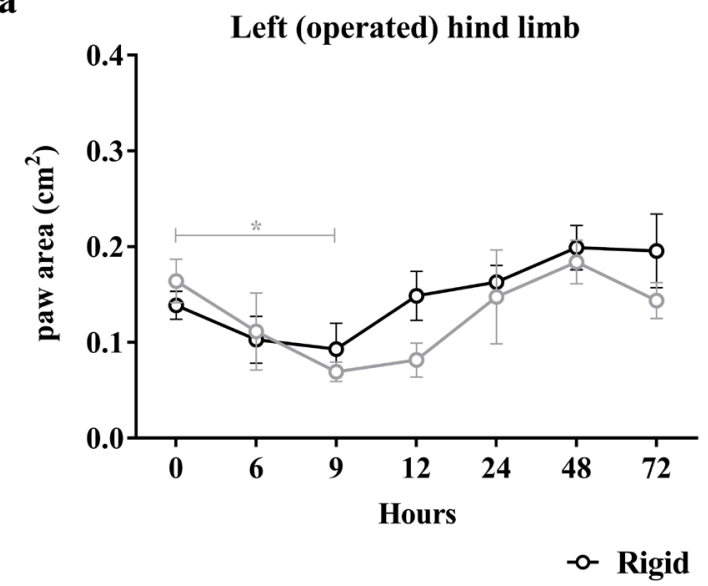

b

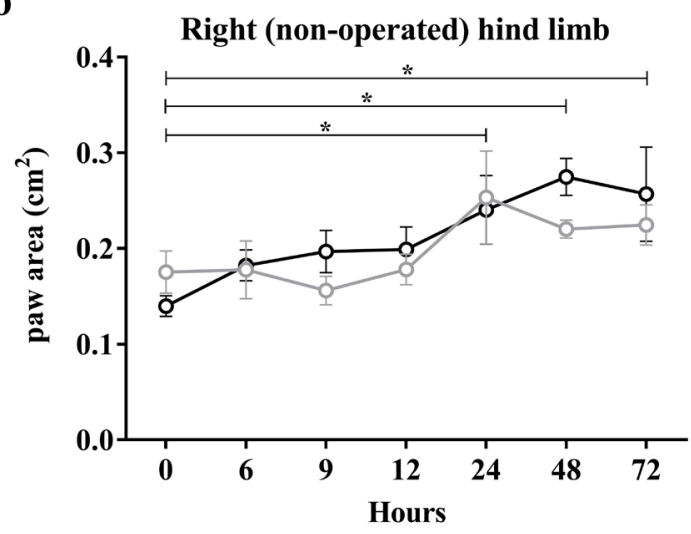

$\sim$ Flexible

Fig. 4. C57BL/6 mice, which received a femoral osteotomy fixed with a rigid or flexible plate, were trained to cross the catwalk and footprints were detected and analysed using the Catwalk XT System. Area $\left(\mathrm{cm}^{2}\right)$ of (a) left and (b) right hind paw footprints was measured. Values were obtained pre-operatively and at $6,9,12,24,48$ and $72 \mathrm{~h}$ post-operatively. Data are shown for animals receiving rigid (black) or flexible (grey) implant $\left(n=5\right.$, mean and SEM; $\left.{ }^{*}=p<0.05\right)$. A reduction in paw area was seen for the operated limb in the flexible group at $9 \mathrm{~h}$, compared to preoperative levels $(p=0.0266)$. The non-operated limb paw area showed an increase over time, being significant for rigid implant group at 24,48 and $72 \mathrm{~h}(p<0.05)$. No significant differences were observed between animals with rigid and flexible implants. 
inoculum are shown in Fig. 5. On day 3, all $S$. epidermidis-inoculated C57BL/6 mice were found to be culture positive for Epi 103.1. The total number of bacteria at day 3 was approximately 10-fold increased relative to the initial inoculated dose. From day 7 onwards, the total CFU count was reduced in all groups and in all compartments (bone, soft tissue and implant) and some mice in both rigid and flexible implant groups were culture-negative in all compartments. Importantly, the proportion of animals that cleared the infection in the rigid implant group $(14 / 28)$ was significantly greater than the number of mice that cleared the infection in the flexible implant group (6/28), when all data points were considered ( $p=0.049$, Fisher exact test, 2 -tailed). The quantitative bacteriology data revealed that mean CFU counts were often higher in mice with flexible

a

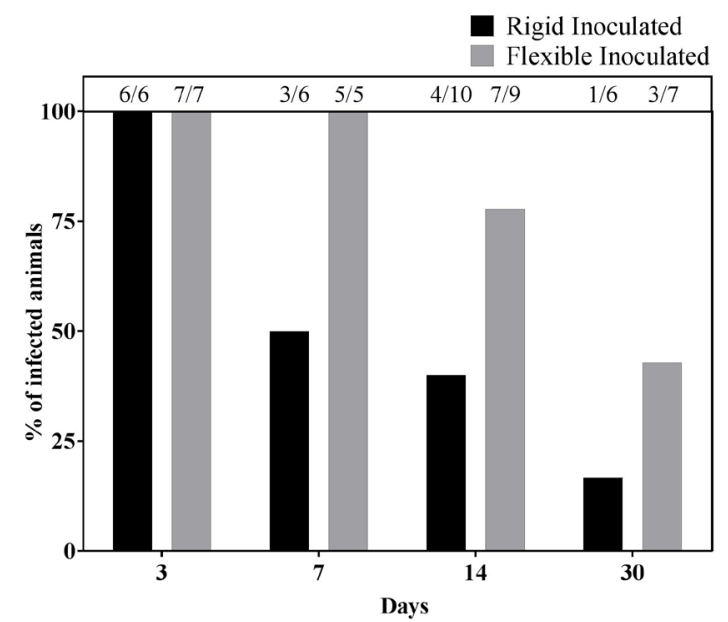

c: Soft tissue

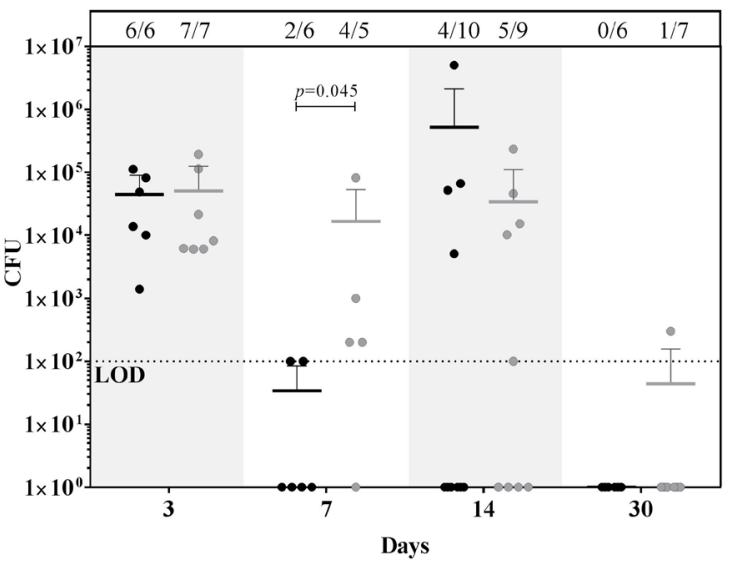

implants, although this only reached statistical significance in soft tissue at day 7 (Fig. 5c).

Results for BALB/c mice inoculated with $S$. epidermidis are shown in Fig. 6. All BALB/c mice were infected 7 and $14 \mathrm{~d}$ post-operatively and CFU counts were 10- to 100-fold higher in bone and implant samples compared to equivalent measurements for C57BL/6 mice (significant differences between total CFU counts at day 7, Kruskal Wallis test with Dunn's post hoc: $p=0.0093)$. Clearance of the infection in $\mathrm{BALB} / \mathrm{c}$ mice was first observed at day 30, with no differences observed between the rigid and flexible groups. With all time points included, the proportion of mice that cleared the bacterial infection in the rigid implant group (4/17) did not differ from the flexible group (3/18). Similarly, there were no differences in CFU counts between mice with flexible or rigid

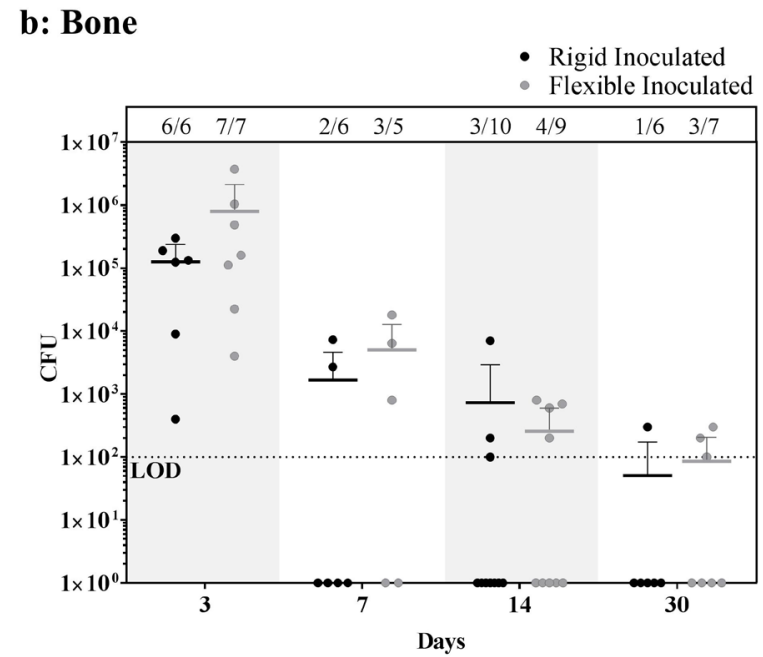

\section{d: Implant}

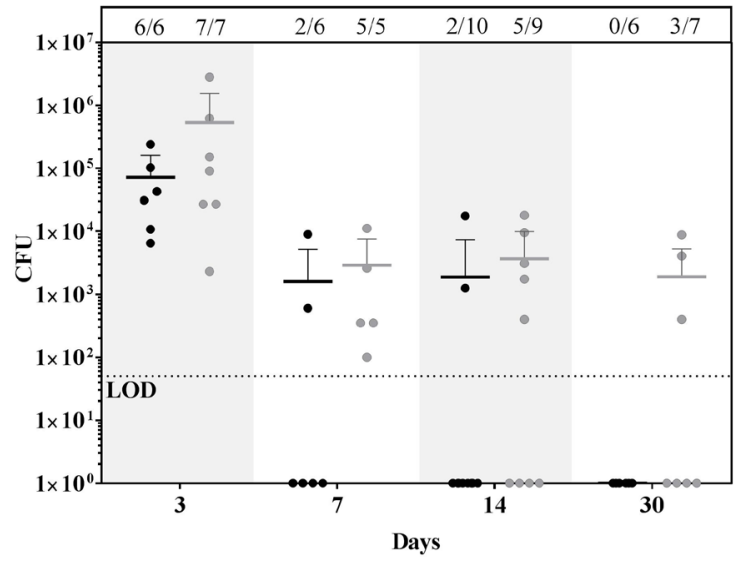

Fig. 5. Effect of fracture fixation mechanical properties in infection progression. C57BL/6 mouse femora were fixed with MouseFix ${ }^{\mathrm{TM}}$ rigid or flexible implants and, after creating a bone defect, $1 \times 10^{4}$ S. epidermidis CFU were inoculated into the osteotomy site. CFU counts were obtained at each time point after tissue homogenisation or implant sonication and posterior plating in BA plates. Results are presented as percentage of $S$. epidermidis-infected C57BL/6 mice at (a) each time point and quantitative bacteriology from each compartment (b, bone; $\mathbf{c}$, soft tissue; $\mathbf{d}$, implant). Additionally, the upper panel of each graph shows proportion of culture positive animals/total animals per group ( $n=5-10$, mean and SD). LOD $=$ limit of detection $\left(1 \times 10^{2} \mathrm{CFU}\right.$ for bone and soft tissue, $0.5 \times 10^{2} \mathrm{CFU}$ for the implant), samples below LOD were represented as 1 . At all time points, animals with a rigid device cleared infection (culture negative for all samples) at a higher percentage, when compared to flexible group. 
implants, except at day 14, when significantly higher levels of bacteria were detected in the soft tissue of the flexible implant group (Fig. 6c).

In the $S$. aureus study, $100 \%$ of C57BL/6 mice from both groups were found infected at all time points at the standard dose (Fig. 7). The number of bacteria cultured from the tissues and the implant on day 3 was 2-3 orders of magnitude higher than CFU counts from S. epidermidis-infected mice. At day 7 and 14, the mean number of bacteria decreased in all compartments in both implant groups; however, it maintained an average CFU burden of $10^{6}-10^{7} \mathrm{CFU}$ per sample, with no clearance of bacteria being observed. In addition, the mean CFU counts between the rigid and flexible implant groups were very similar across the experiment. At all time points, S. aureus total CFU counts were significantly higher compared to

a

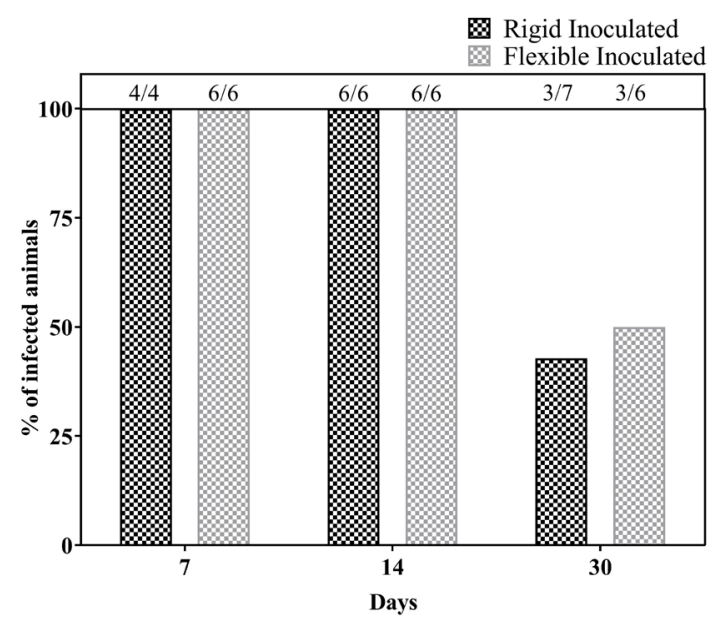

c: Soft tissue

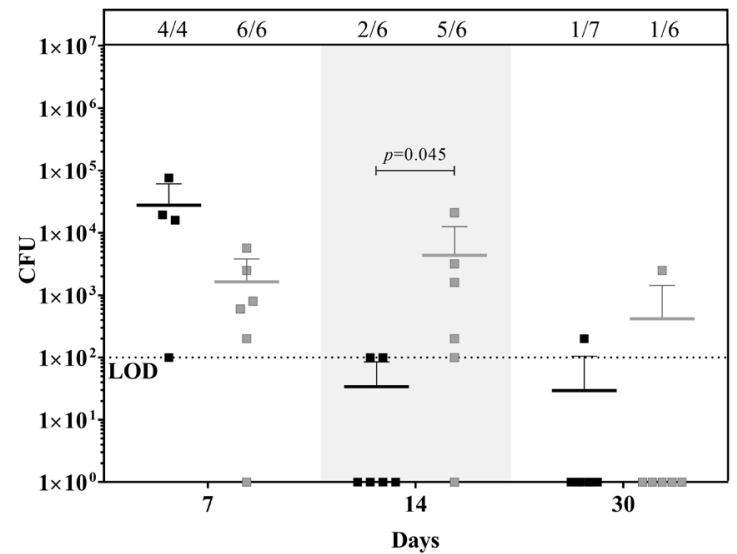

S. epidermidis total CFU counts, in C57BL/6 mice (Kruskal-Wallis test with Dunn's post hoc: $p<0.0001$ ). Since no clearance was observed in C57BL/6 mice and severe osteolysis and tissue damage were observed in the animals, this experiment was not performed in BALB/c mice.

As the initial S. aureus inoculum dose resulted in a $100 \%$ infection rate for the entire study period, no impact of fixation type on infection was identified. Thus, the experiment was repeated at a lower dose in both mice strains, where the animals were expected to have a better chance of clearing the infection and a role for fracture stability might have emerged. However, even when the initial dose was reduced 10-fold, neither mouse strain cleared the infection by day 14 and CFU counts were similar to those measured for the $S$. aureus standard dose group (Fig.

\section{b: Bone}

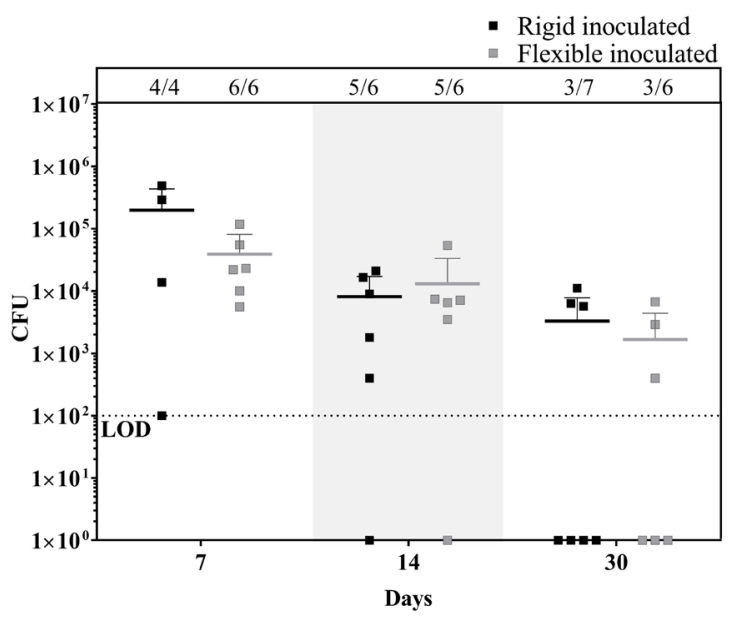

d: Implant

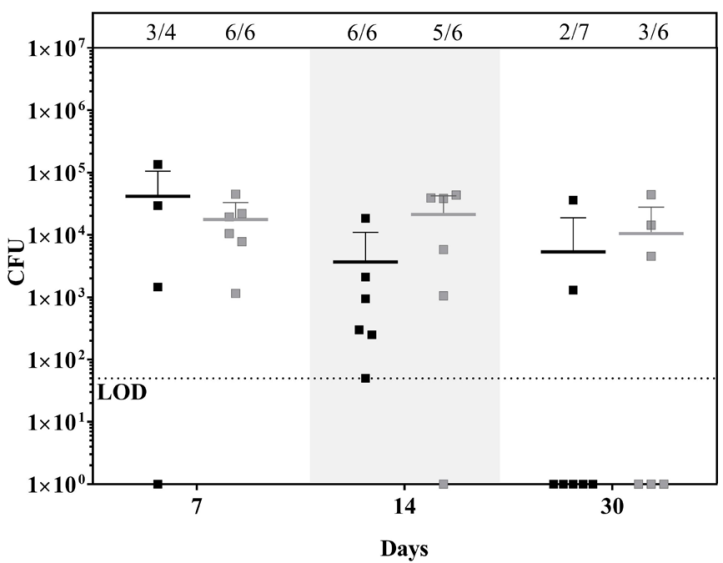

Fig. 6. Effect of fracture fixation mechanical properties on infection progression. BALB/c mouse femora were fixed with MouseFix ${ }^{\mathrm{TM}}$ rigid or flexible implants and, after creating a bone defect, $1 \times 10^{4}$ S. epidermidis CFU were inoculated into the osteotomy site. CFU counts were obtained at each time point after tissue homogenisation or implant sonication and posterior plating in BA plates. Results are presented as percentage of $S$. epidermidis infected BALB/c mice at (a) each time point and quantitative bacteriology from each compartment (b, bone; $\mathbf{c}$, soft tissue; $\mathbf{d}$, implant). Additionally, the upper panel of each graph shows proportion of culture positive animals/total animals per group ( $n=4-7$, mean and SD). LOD = limit of detection $\left(1 \times 10^{2} \mathrm{CFU}\right.$ for bone and soft tissue, $0.5 \times 10^{2} \mathrm{CFU}$ for the implant), samples below LOD were represented as 1 . It could be observed that contrary to C57BL/6 mice, BALB/c did not clear infection at 7 or $14 \mathrm{~d}$ post-operatively, irrespectively of implant type. At day 30, mice from both implant groups cleared the infection. 
8). Once more, purulent tissue and osteolytic regions were observed, with no complete closure of the gap. Despite the initial inoculum being lower, total $S$. aureus CFU counts in those animals were significantly higher compared to $S$. epidermidis total CFU counts in both C57BL/6 and BALB/c mice at day 14 (MannWhitney test, $p<0.0001$ for both comparisons).

\section{Spleen and popliteal lymph node cell counts}

Total cell counts were performed in lymph node and spleen single cell suspensions to evaluate inflammation in local and systemic secondary lymphoid organs, respectively. The type of fixation did not have an impact upon cell counts from spleens or popliteal lymph nodes of C57BL/6 mice either with or without S. epidermidis inoculation (Fig. 9). Levels were only slightly increased at day 3 compared to day 0 , probably due to inflammation caused by surgical

$\mathbf{a}$

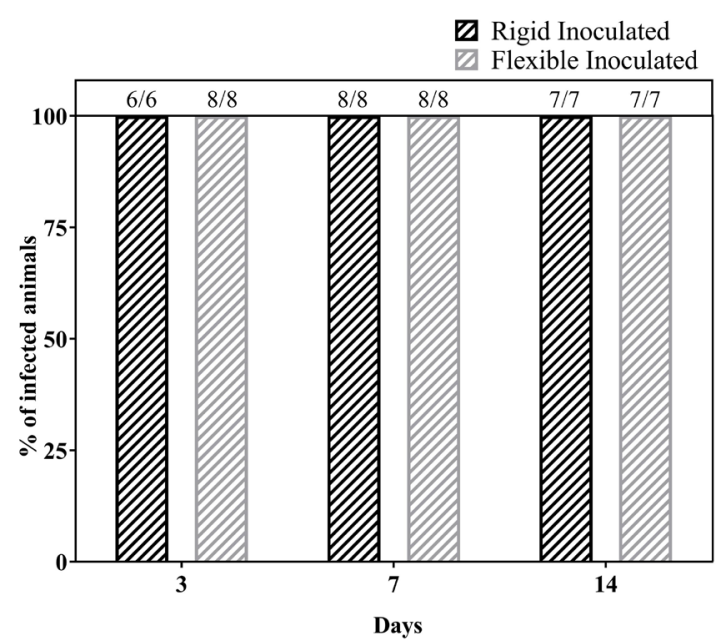

c: Soft tissue

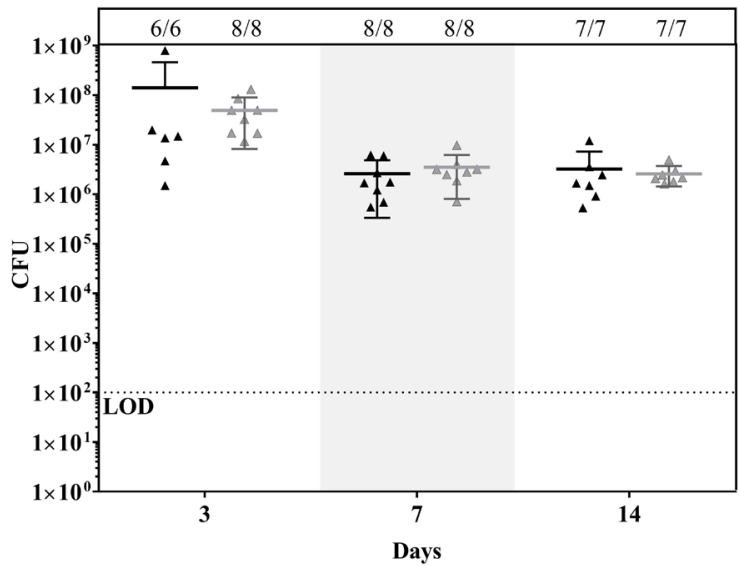

procedure, but they returned to baseline levels afterwards. S. aureus infection resulted in a significant increase in spleen and lymph node cell counts at day 7 and 14, when compared to the non-inoculated or S. epidermidis-inoculated equivalents for both rigid and flexible implant groups (Fig. 9a,b). Finally, cell counts in the bone samples did not show significant differences (data not shown).

In BALB/c mice, S. epidermidis-inoculated animals showed an increase in cell numbers at day 7 in spleen and lymph node, compared to non-inoculated animals (Fig. 9c,d). This larger number of cells in inoculated animals may be linked to the higher percentage of infected animals and higher CFU counts in BALB/c mice compared to C57BL/6. Levels returned to baseline values from day 14 . For femur cell counts, no significant differences among the groups were observed with regards to implant type or

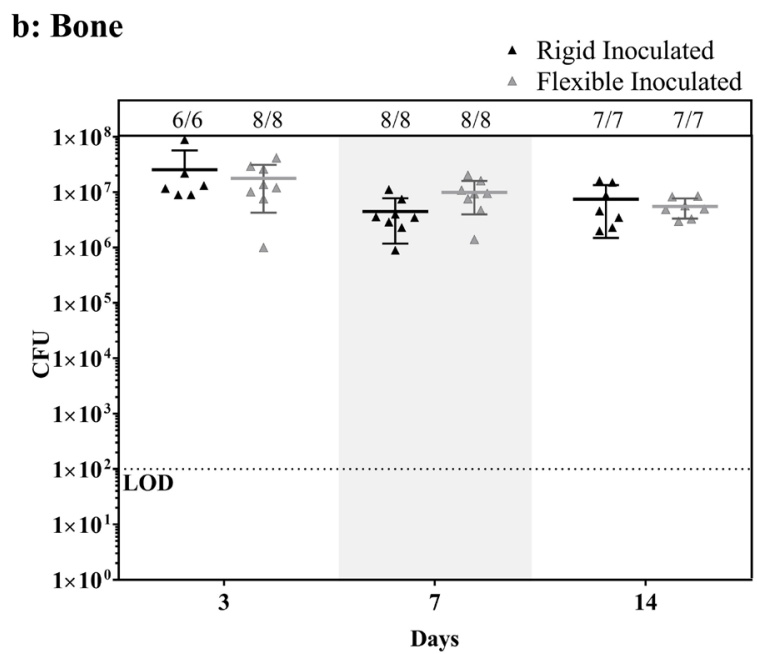

d: Implant

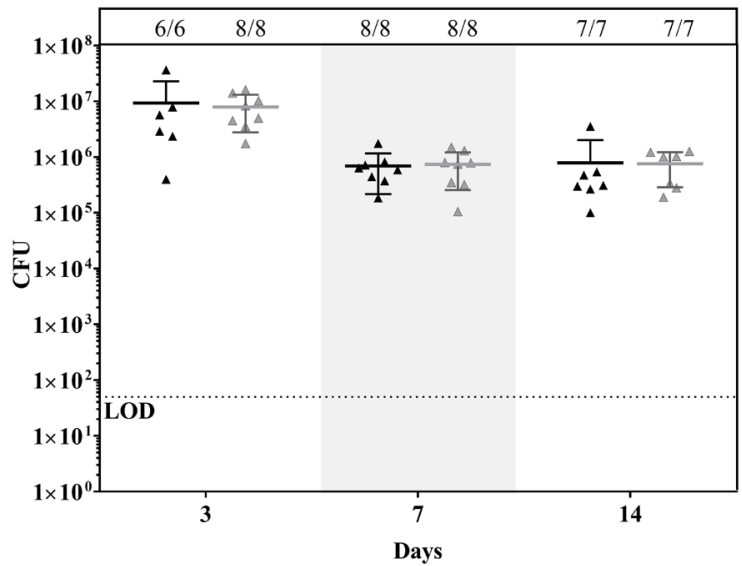

Fig. 7. Effect of fracture fixation mechanical properties on infection progression. C57BL/6 mouse femora were fixed with MouseFix ${ }^{\mathrm{TM}}$ rigid or flexible implants and, after creating a bone defect, $1 \times 10^{4} \mathrm{~S}$. aureus CFU were inoculated into the osteotomy site. CFU counts were obtained at each time point after tissue homogenisation or implant sonication and posterior plating in BA plates. Results are presented as percentage of $S$. aureus infected C57BL/6 mice at (a) each time point and quantitative bacteriology from each compartment (b, bone; $\mathbf{c}$, soft tissue; $\mathbf{d}$, implant). Additionally, the upper panel of each graph shows proportion of culture positive animals/total animals per group $(n=6-8$, mean and SD). LOD $=$ limit of detection $\left(1 \times 10^{2} \mathrm{CFU}\right.$ for bone and soft tissue, $0.5 \times 10^{2} \mathrm{CFU}$ for the implant), samples below LOD were represented as 1 . As shown, no animal cleared bacteria at any time point. In addition, high bacterial counts were recovered from all compartments, being significantly higher compared to S. epidermidis counts in C57BL/6. 
among non-inoculated and S. epidermidis-inoculated animals.

\section{Histological evaluation}

Additional animals were used to obtain intact soft tissue and femurs with implant in place for histological analysis (Fig. 1). Representative overview images of the histological features of C57BL/6 mice with rigid and flexible implants at day 14 and 30 are shown in Fig. 10 (for non-inoculated mice) and Fig. 11 (for inoculated mice).

Non-inoculated mice with rigid implant fixation were observed to undergo a mixture of intramembranous and endochondral ossification at day 14 (Fig. 10a,b), with the osteotomy gap filled with new bone (Fig. 10b). At the same time point, noninoculated mice fixed with the flexible implant were undergoing endochondral ossification with a large stabilising, mainly cartilaginous, callus (Fig. 10e,f, white asterisk: cartilaginous callus). The osteotomy gap in these mice was filled mostly with fibrin and

$\mathbf{a}$

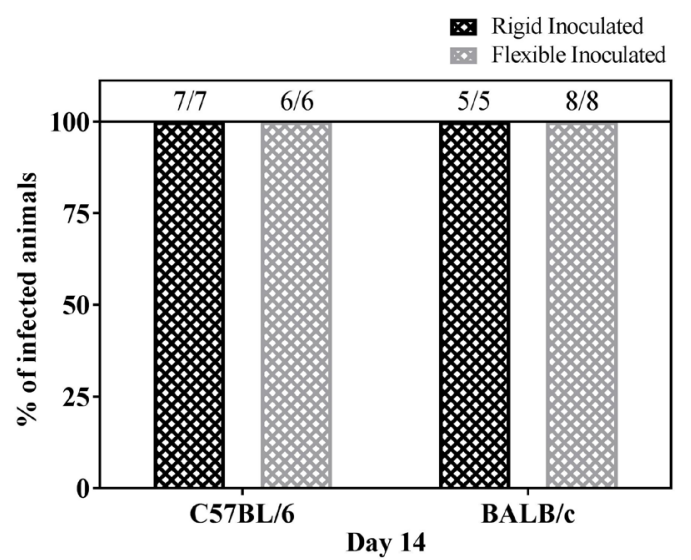

c: Soft tissue

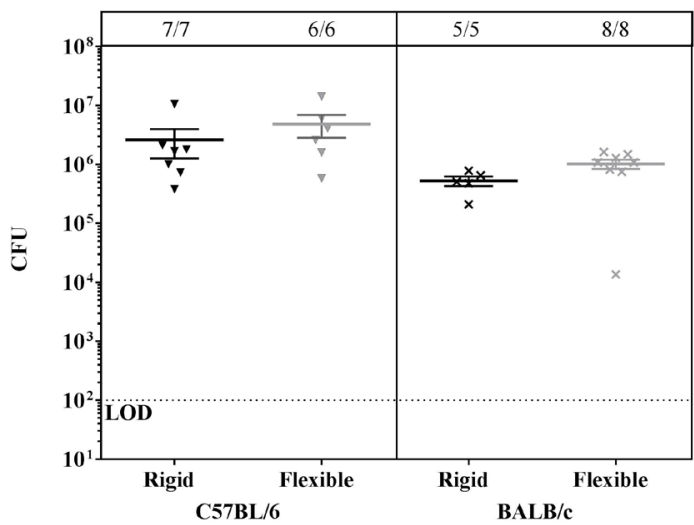

cartilage (Fig. 10f) and a region of fibrous tissue was observed in the bone marrow, in the distal side of the osteotomy (Fig. 10f, black asterisk). Early granulation tissue, as well as muscle regeneration, was observed over the screw heads and implant of both groups, often more obvious in flexible group (Fig. 10c,d for rigid and $\mathbf{g}, \mathbf{h}$ for flexible, black arrows: blood vessels).

At day 30, the osteotomy gap was completely closed in animals with a rigid implant (Fig. 10j, black arrow), while the gap was still visible in most of the animals with a flexible implant (Fig. 10n, black arrow), suggesting a delay in bone healing in the flexible group. The large cartilaginous callus observed at day 14 was under remodelling by day 30 , consisting predominantly of woven bone and secondary bone marrow (Fig. 10m,n, black asterisk). The granulation tissue overlying the plate at that time point was only a thin layer of fibrous tissue (Fig. $10 \mathbf{k}, \mathbf{l}, \mathbf{o}, \mathbf{p}$, white asterisk).

S. epidermidis-inoculated animals are shown in Fig. 11. Inoculation did not seem to impair the

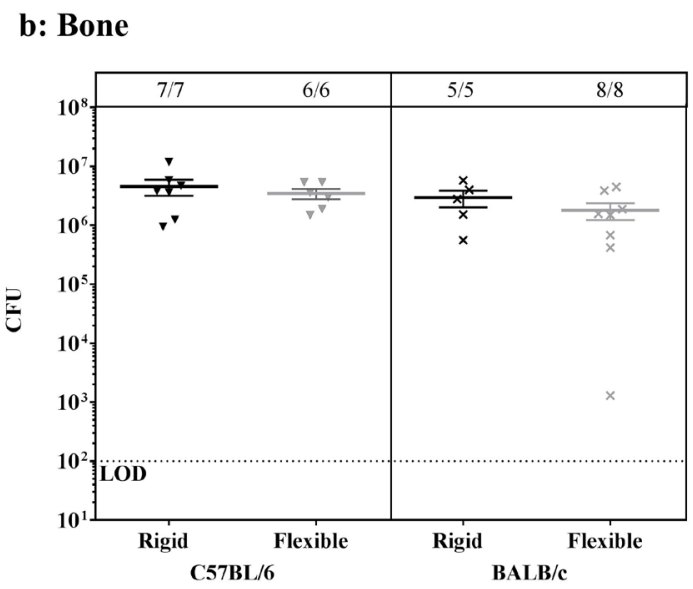

d: Implant

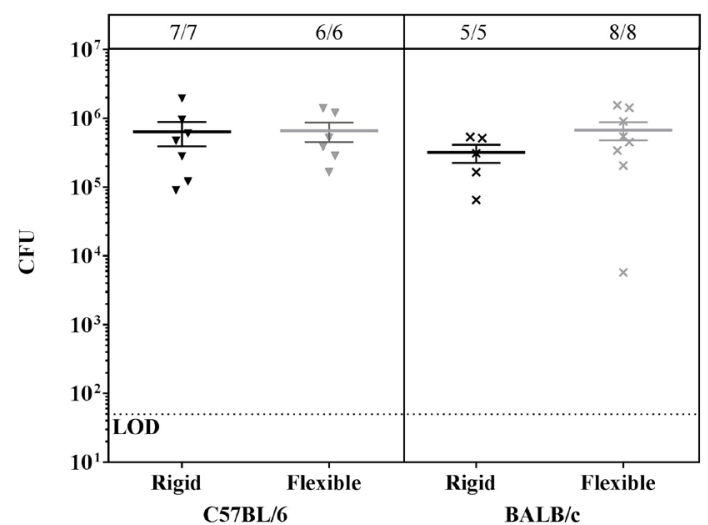

Fig. 8. Effect of fracture fixation mechanical properties in infection progression. C57BL/6 and BALB/C mouse femora were fixed with MouseFix ${ }^{\mathrm{TM}}$ rigid or flexible implants and, after creating a bone defect, $1 \times 10^{3} \mathrm{~S}$. aureus $\mathrm{CFU}$ were inoculated into the osteotomy site. CFU counts were obtained at each time point after tissue homogenisation or implant sonication and posterior plating in BA plates. Results are presented as percentage of low dose $S$. aureus infected C57BL/6 and BALB/c mice at (a) day 14 and quantitative bacteriology from each compartment (b, bone; $\mathbf{c}$, soft tissue; $\mathbf{d}$, implant). Additionally, the upper panel of each graph shows proportion of culture positive animals/total animals per group $(n=5-8$, mean and $\mathrm{SD})$. $\mathrm{LOD}=$ limit of detection $\left(1 \times 10^{2} \mathrm{CFU}\right.$ for bone and soft tissue, $0.5 \times 10^{2} \mathrm{CFU}$ for the implant $)$, samples below LOD were represented as 1 . Despite using a 10-times lower dose compared to standard inoculum, all animals remained infected, with high bacterial counts in all compartments. 
progression of bone healing at day 14 in either of the implant groups, when compared to non-inoculated equivalents (Fig. 11a,b,e,f), with new bone formation in the osteotomy of animals with a rigid implant and a big cartilaginous callus in the flexible implant group. At day 14, more soft tissue inflammation was observed in inoculated animals, as reflected in a greater lymphocyte and granulocyte infiltration in surrounding soft tissue and the presence of fluidfilled spaces (Fig. 11a,c-e,g,h, black arrows). Such signs were often localised into small foci and not widespread throughout the tissues. In some cases, osteolysis around screws was observed (Fig. 11a,b, white arrows), with more granulocytic infiltrate compared to the aseptic osteolysis that occurred in some non-inoculated animals due to instability or stress shielding (Fig. 12).

By day 30, the osteotomy was mostly closed in inoculated animals with a rigid implant, while in mice with a flexible implant the cartilaginous callus was in a remodelling phase. The soft tissue showed a similar appearance to the non-inoculated animals (Fig. 11k,l,o,p). Signs of infection, such as higher bone remodelling, indicated by larger regions with secondary bone marrow (Fig. 11i,m), were found. Osteolysis was observed in some inoculated animals, with some granulocyte infiltrates. However, these were not general features and evidence of infection was weak or even absent in both implant groups, supporting the microbiology data indicating that many of the animals had cleared the infection by this time point.

In BALB/c mice, the histological observations regarding bone healing were similar (data not shown), with often earlier closure in animals with a rigid implant and a cartilaginous callus and a remodelling phase at day 30 in animals with a flexible implant. Interestingly, a more inconsistent healing was detected in this strain, with some animals showing no gap closure at all at day 14 . To our knowledge, this is the first study using flexible MouseFix ${ }^{\mathrm{TM}}$ plate in $\mathrm{BALB} / \mathrm{c}$ background. Importantly, this phenomenon was not linked to the presence of bacteria. Signs of infection were also similar to that observed for C57BL/6 mice, only being evident in soft tissue over the plate at day 14 and becoming less pronounced at day 30.

Bacteria were observed on the implants from C57BL/6 and BALB/c mice at day 14 and also in paraffin-embedded sections (Fig. 13).

\section{Discussion}

Despite the prevalence of S. epidermidis and other CoNS in orthopaedic implant-related infections, the scientific literature in the field is dominated by S. aureus (Lovati et al., 2017). To the best of our a: Spleen

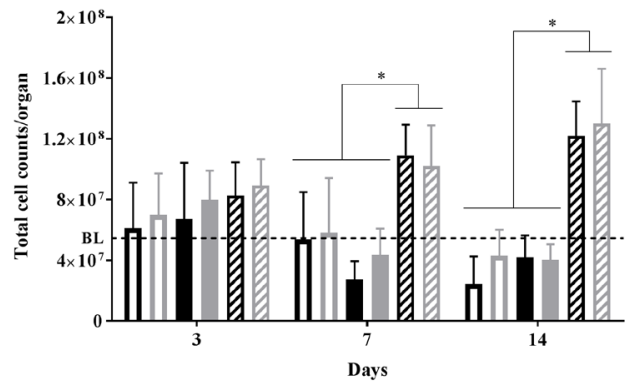

c: Spleen

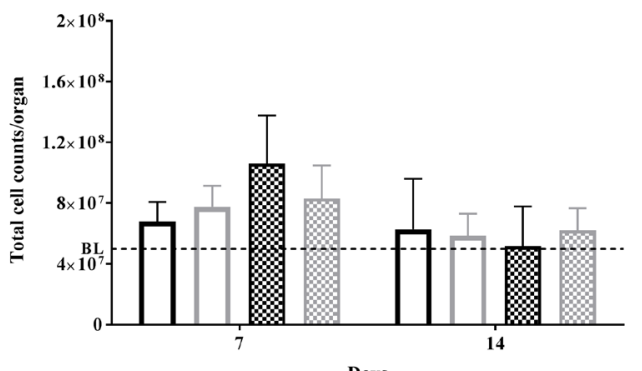

Days b: Popliteal lymph node

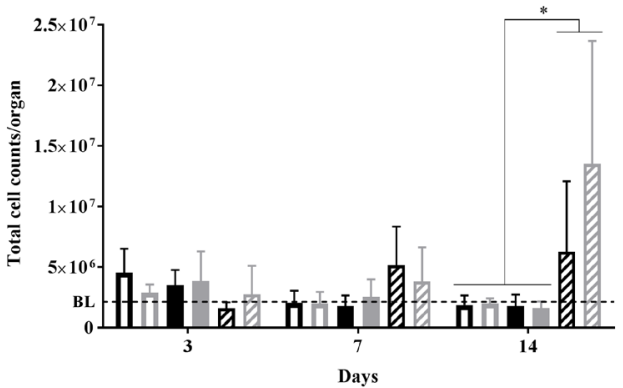

Non-Inoculated Rigic

S. epidermidis Inoculated Rigid
S. epidermidis Inoculated Flexibl

S. aureus Inoculated Rigid S. aurens Inoculated Flexible

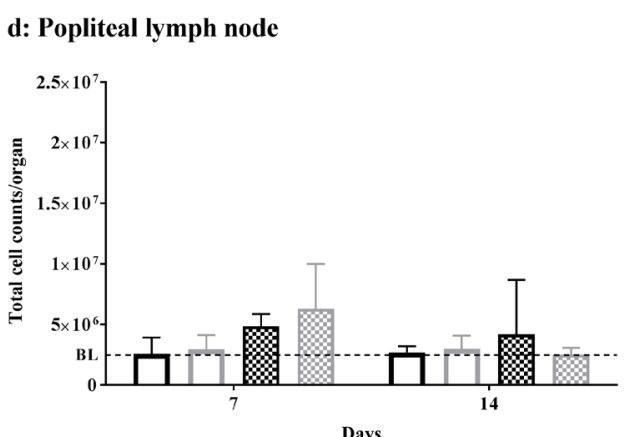

Fig. 9. Total cell counts in spleen and popliteal lymph node of C57BL/6 and BALB/c mice. Spleen and popliteal lymph node were mashed and filtered in order to obtain single cell suspensions. Figure depicts total counts in spleen and popliteal lymph node of $(\mathbf{a}, \mathbf{b})$ C57BL/6 and $(\mathbf{c}, \mathbf{d})$ BALB/c mice with a rigid (black) or flexible (grey) implant at 3, 7 and $14 \mathrm{~d}$. BL (dashed line): baseline levels from average values of control animals $\left(n=4-10\right.$, mean and SEM; $\left.{ }^{*}=p<0.05\right)$. All operated animals presented an early increase in spleen and lymph node cell counts, probably due to the inflammation induced by surgical procedure. This increase was not significant compared to baseline levels. At later time points, only S. aureus-inoculated animals (C57BL/6 mice) presented significantly larger cell counts, supporting a more severe infection process and higher inflammation process. 
knowledge, there is only one model described for S. epidermidis FRI (Lovati et al., 2016a; Lovati et al., $2016 \mathrm{~b})$, in rats. In the present study, we established and characterised an S. epidermidis FRI model in mice and further developed the model to study the influence of fracture stability on infection progression. To overcome the limitations of previous preclinical in vivo models assessing the influence of stability on infection (Friedrich and Klaue, 1977; Merritt and Dowd, 1987; Rittman and Perren, 1974; Worlock et al., 1994), we used a standardised femoral fixation system previously described for monitoring fracture healing under different biomechanical conditions (Grongroft et al., 2009; Steck et al., 2011). Using gait analysis, we showed that both rigid and flexible fixation allowed the mice to return to baseline mobility within a short period after surgery and histological observations confirmed healing to proceed under mostly intramembranous ossification and endochondral ossification respectively, as expected for the implant fixation used. In terms of the clinical appearance of the infections in this study and their relevance to clinical FRI in humans, S. epidermidis-inoculated mice (both mice strains) did not show major differences over time in weight change or cell counts, suggesting a subclinical type

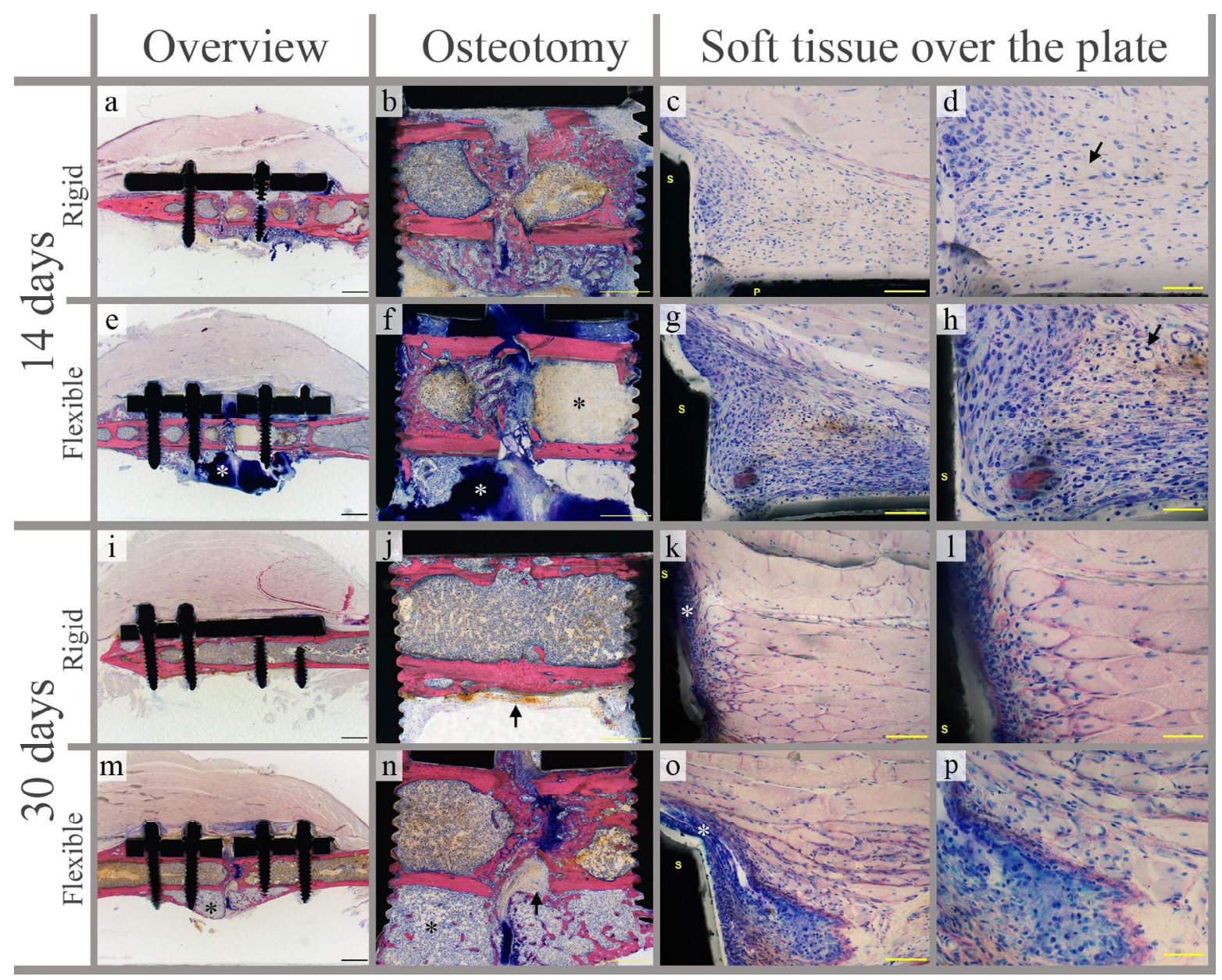

Fig. 10. Light microscope images of GE-stained PMMA sections of non-inoculated C57BL/6 mice at day 14 and 30 fixed with rigid and flexible implants. First column: overview images of femur with implant in place including the surrounding soft tissue (scale bar: $1 \mathrm{~mm}$ ). Second column: higher-magnification image of the osteotomy site (scale bar: $500 \mu \mathrm{m}$ ). Third column: tissue overlying the plate (scale bar: $200 \mu \mathrm{m})$. Fourth column: higher magnification of the overlying soft tissue (scale bar: $100 \mu \mathrm{m})$. Screw (S) and plate $(\mathrm{P})$ are annotated in high magnification images to aid orientation. At day 14, the rigid implant group (a) showed signs of new periosteal and endosteal bone closing the osteotomy site (b), while flexible implant group (e) at the same time point showed a big cartilaginous callus (e,f, white asterisk) and a lack of closure of the osteotomy site. Of note, the bone marrow at the distal side of the osteotomy appeared unstained in the flexible group, consisting of fibrotic tissue (f, black asterisk). Both flexible and rigid groups displayed early granulation tissue $(\mathbf{c}, \mathbf{d}, \mathbf{g}, \mathbf{h})$ above the plate consisting of fibroblasts, new vessels (d,h, black arrows) and leukocyte infiltrate. At day 30, in mice fixed with rigid implants (i) the osteotomy was closed (j, black arrow). In the flexible implant group $(\mathbf{m})$ the callus was still remodelling $(\mathbf{m}, \mathbf{n}$, black asterisk), with substantial amounts of cartilage tissue present and full closure of the osteotomy was not achieved (n, black arrow). The inflammation of the tissue over the plate was reduced to a thin layer of fibrous tissue (k,o, white asterisk), particularly in the rigid implant group. 
of infection. This closely matched the typical clinical picture of S. epidermidis infection (Metsemakers et al., 2016; Schafer et al., 2008). Instead, animals infected with $S$. aureus exhibited greater weight loss, larger CFU counts in tissues and greater cell numbers in spleen and lymph node, suggesting the recruitment of lymphocytes and leukocytes, and overall more inflammation. In addition, purulent tissue was observed over the plate and osteolysis was observed around the screws, below the implant and at the osteotomy site. This also correlated with clinical observations, whereby S. aureus infection is associated with acute onset of symptoms (Metsemakers et al., 2016).
S. epidermidis infection resolved in many animals, which was similar to previous implant-associated infection models for this organism (Lankinen et al., 2012; Laure et al., 2008; Lovati et al., 2016b). Higher dose inocula may guarantee a persistent infection; however, as reported recently for S. aureus, infectious dose might alter immune responses and has to be considered carefully (Vidlak and Kielian, 2016). Considering our experimental hypothesis that fracture instability predisposes to infection, a low dose inoculum enabled us to differentiate between the development of infection (or not) rather than merely looking for changes in CFU counts, which would be less indicative of an effect. Of note, even

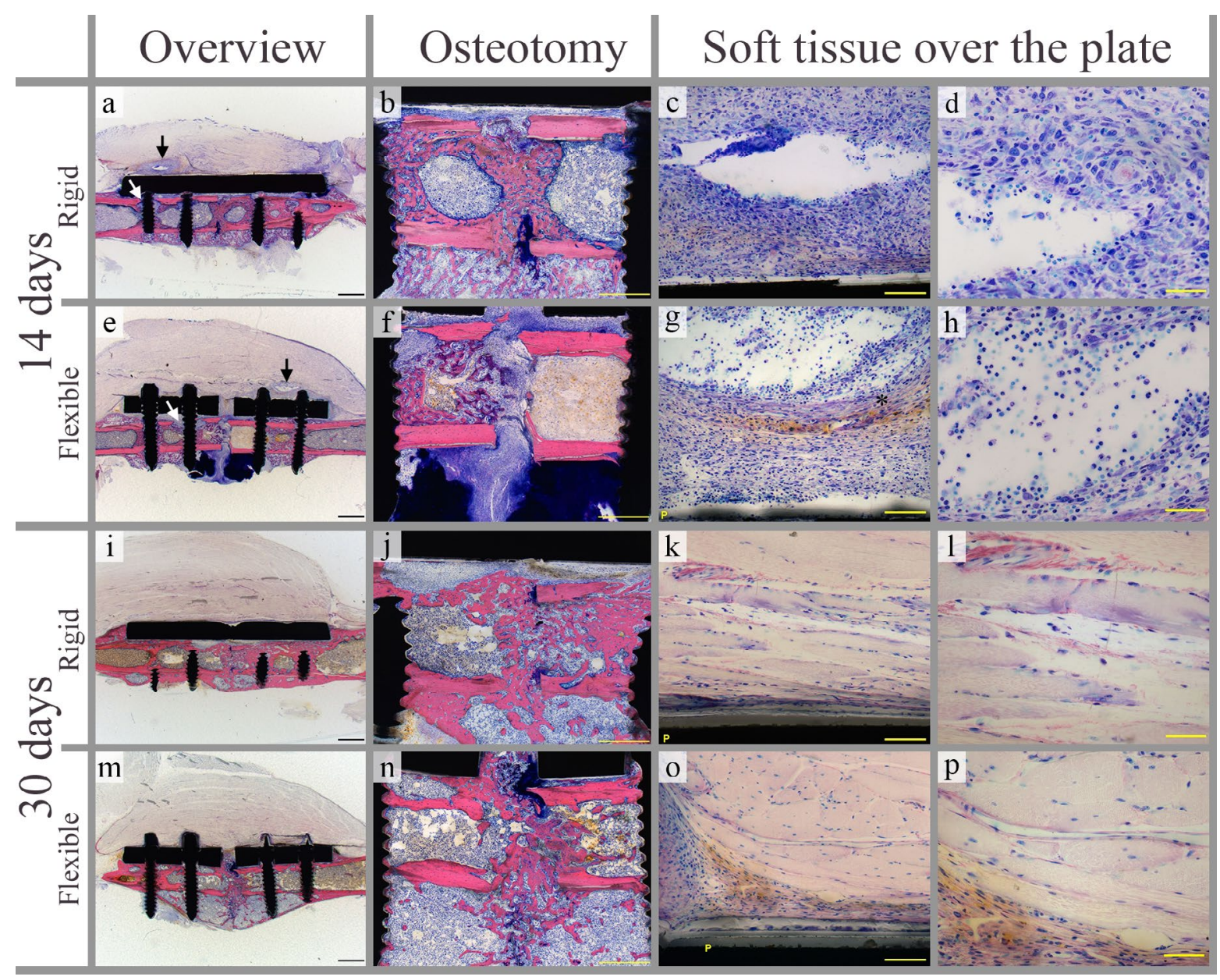

Fig. 11. Light microscope images of GE-stained PMMA sections of S. epidermidis-inoculated C57BL/6 mice at day 14 and 30 fixed with rigid and flexible implants. First column: overview images of femur with implant in place including the surrounding soft tissue (scale bar: $1 \mathrm{~mm}$ ). Second column: higher magnification image of osteotomy site (scale bar: $500 \mu \mathrm{m})$. Third column: tissue overlying the plate (scale bar: $200 \mu \mathrm{m})$. Fourth column: higher magnification of the overlying soft tissue (scale bar: $100 \mu \mathrm{m})$. Plate $(\mathrm{P})$ is annotated in high magnification images to aid orientation. At day 14, once again, the osteotomy site in the rigid group showed a high degree of closure $(\mathbf{a}, \mathbf{b})$, while a complete non-closure of the osteotomy site and a large cartilaginous callus was observed in the flexible group (e,f). In both implant groups, small foci of inflammatory cell accumulation (a,e, black arrow) were seen in the soft tissue. These foci, with high infiltration of polymorphonuclear cells, are shown in high magnification images $(\mathbf{c}, \mathbf{d}, \mathbf{g}, \mathbf{h})$. In some cases, the presence of a fibrous capsule was observed (g, black asterisk), suggesting that this focus could be defined as a small encapsulated abscess. The bone edges at the osteotomy site and near the screws showed signs of osteolysis (a,e, white arrows). At day 30, closure of the osteotomy site was advanced in both rigid and flexible implant groups. In contrast to day 14, inflammation of the tissue above the plate had receded substantially $(\mathbf{k}, \mathbf{l}, \mathbf{o}, \mathbf{p})$. 
when using a low dose inoculum, infection persisted in some animals for up to $30 \mathrm{~d}$. It was challenging to detect bacteria in the histological samples, which would have been interesting in order to determine the localisation of the bacteria in this model. Some biofilm-like structures were observed on the surface of the implants in the SEM images and located bacteria in locations of difficult access, such as filling bone vessels within the bone (Fig. 13). In addition, $S$. epidermidis can persist intracellularly in vitro (Magrys et al., 2015) and in vivo (Boelens et al., 2000; Riool et al., 2014), although intracellular bacteria were not specifically searched for in the samples.

In testing the role of fracture stability on infection progression under controlled conditions, the desire was to advance the field from the data provided by early studies (Friedrich and Klaue, 1977; Merritt and Dowd, 1987; Rittman and Perren, 1974; Worlock et al., 1994). Friederich and Klaue (1977) show that stable fractures are associated with a lower S. aureus infection rate in rabbits. However, in this study, animals are allocated to the stable or unstable group after radiographic observations, with both plate and nail fixation methods present in both study groups, as pre-allocation based upon implant type is evidently unable to provide predictable mechanics. In 1987, Merritt et al. performed a study with open fractures in hamsters (Merritt and Dowd, 1987); however, they compare a K-wire-fixed fracture with a non-fixed fracture having no foreign body in the study, which thereby misses an important component of a device-associated infection, i.e. the device itself. Finally, in the most recent experimental study on this topic by Worlock et al. (1994), the stable construct (plate) shows a lower $S$. aureus infection rate compared with the unstable construct (an intramedullary rod). Therefore, once again, this study has a confounding factor of whether different fixation systems (plate vs. intramedullary rod) may also have influenced the infection rates. The current study provided the first standardised model, allowing for controlled conditions. Two similar implant types, with validated different mechanical properties and reliable healing outcomes, were successfully used, confirming previous observations made in C57BL/6 mice regarding bone healing (Grongroft et al., 2009;

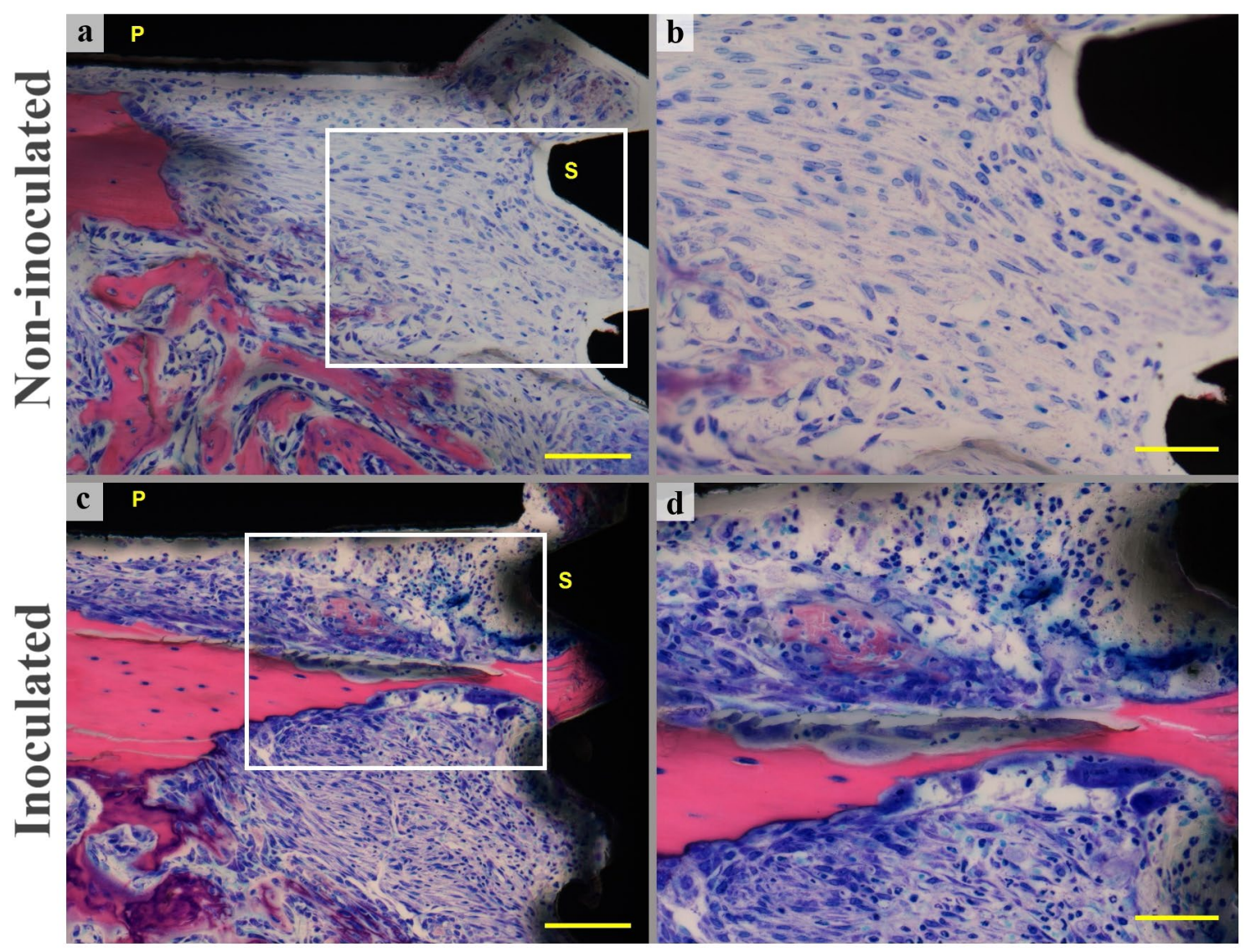

Fig. 12. Osteolytic regions observed in non-inoculated and S. epidermidis-inoculated mice. Light microscope images of GE-stained thick sections at day 14 in C57BL/6 mice with a rigid implant (non-inoculated and S. epidermidis-inoculated). N.B. small areas of low osteointegration near screws in both groups. In non-inoculated animals, new bone formation (callus) was observed and the tissue primarily consisted of fibroblasts/fibrocytes, small number of capillaries and very few mononuclear inflammatory cells (late granulation tissue). In contrast, in inoculated animals, scalloped bone surface and osteoclasts could be observed (osteolysis) and the inflammatory cell infiltration of the granulation tissue was much greater, consisting predominately of polymorphonuclear cells and tissue debris (microabscess). Scale bar: $100 \mu \mathrm{m}$ (first column) and $50 \mu \mathrm{m}$ (second column, magnification images). 
Montjovent et al., 2013; Steck et al., 2011). The two implants were also compared in BALB/c mice, reproducing the same healing outcomes as described before, but in a more inconsistent way, with some animals depicting no closure of the osteotomy site after $14 \mathrm{~d}$. In addition, this model was extended into the FRI field. This study provided evidence that $S$. epidermidis infection could, in fact, be influenced by implant stability. After inoculation with S. epidermidis, the infection clearance over time was enhanced in C57BL/6 mice fixed with rigid plates and bacterial burden displayed a trend towards smaller numbers. This result agreed with all earlier studies on the fact that instability is a risk factor for infection or infection progression. Ongoing work is attempting to provide some understanding of this phenomenon by assessing immune markers in samples obtained in this study. Previous publications focusing on bone healing describe different gene and protein expression patterns between both implant types (Montjovent et al., 2013; Ueno et al., 2011). Therefore, it would be desirable to extend this knowledge by studying samples from infected animals, where the prediction is made that the environment may also differ between both implant types over time. This may lead to the description of local factors that could benefit or hinder bacteria clearance.

The observation that stability influenced $S$. epidermidis infection did not hold true for the second mouse strain. Interestingly, BALB/c mice first achieved clearance of infection at day 30, a time point when the osteotomies in both rigid and flexible groups had already healed. As such, mechanical differences between groups at day 30 may be small or non-existent. In fact, infection clearance was equal between both groups at $30 \mathrm{~d}$, further supporting the need for stability to eradicate infection. Similarly, there was no difference in infection rate between those groups when inoculated with $S$. aureus. It is important to note, however, that $S$. aureus displayed a virulent behaviour in this model, leading to periimplant osteolysis $6 \mathrm{~d}$ post-operatively, as shown previously in a different model (Stadelmann et al., 2015). This osteolysis was observed at both the standard and low doses, making comparison between groups irrelevant, as all animals were effectively unstable. Therefore, these data did not discredit the requirement for implant stability, although the effect may be more pronounced under conditions of a lowgrade infection.
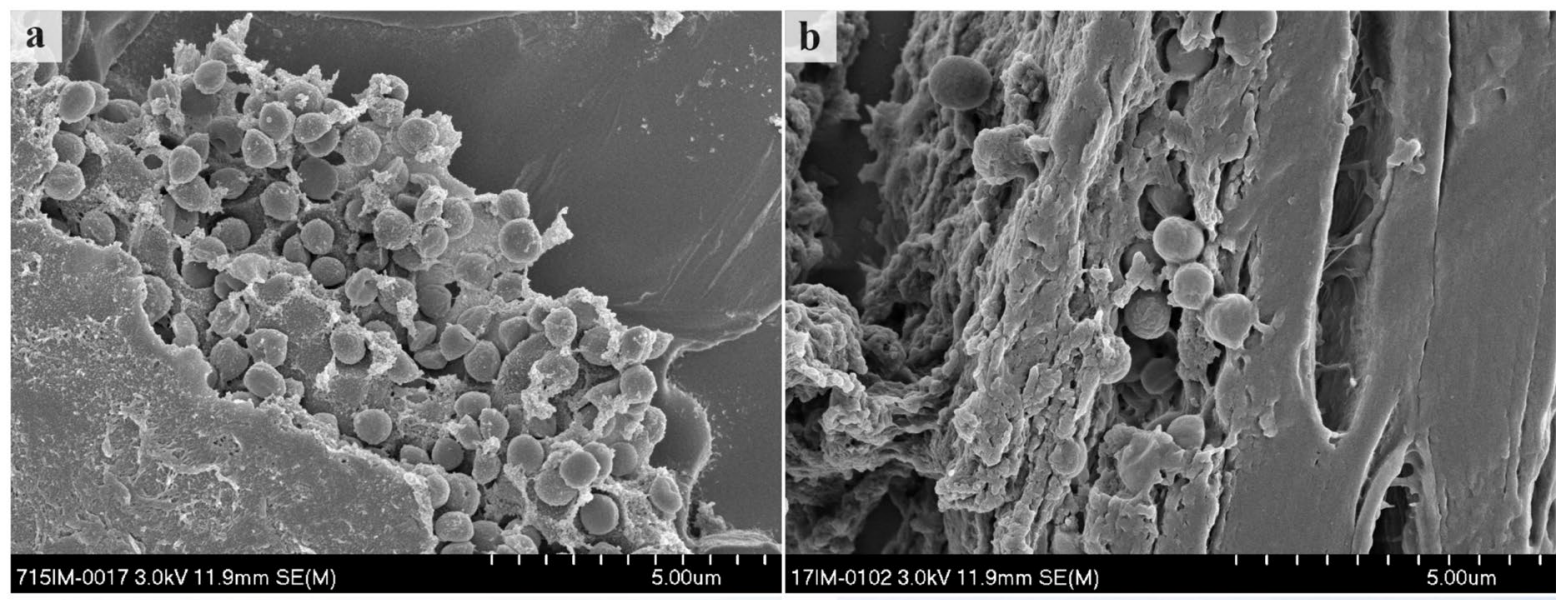

c

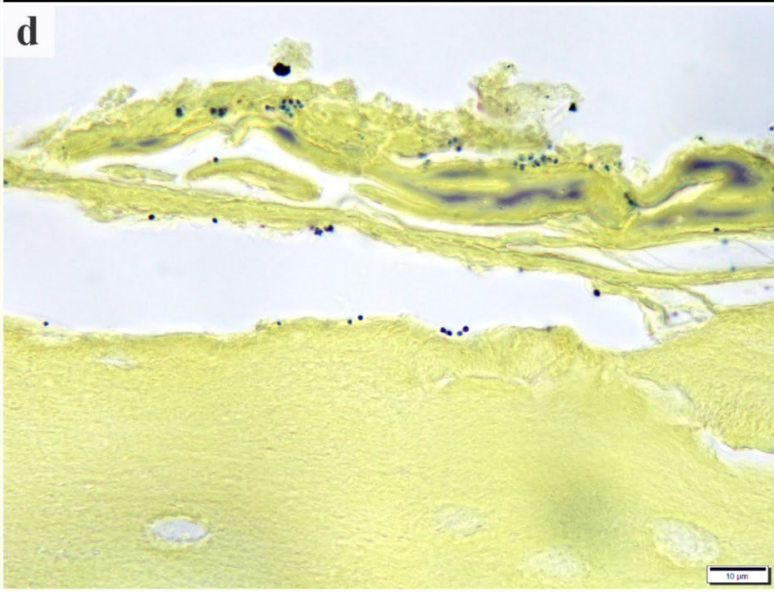

Fig. 13. Bacteria could be observed on the implant and bone in SEM images of gold palladium sputtercoated implants and paraffin sections and in light microscope images of BB-stained paraffin sections. SEM images show clusters of coccoid bacteria attached to (a) the implant surface and (b) in the periosteum. They were also observed in sections stained with BB in (c) a bone vessel or (d) in the periosteum/delaminated bone fragments. Scale bars: (a,b) $5 \mu \mathrm{m}$; (c,d) $10 \mu \mathrm{m}$. 
Patient factors, such as age or co-morbidities, are known to influence infection risk and need to be considered for treatment management (Forsberg et al., 2011; Marmor and Kerroumi, 2016; Willey and Karam, 2016). In the current study, when using two different inbred strains, C57BL/6 mice were observed to clear bacteria more rapidly and presented lower CFU counts at most of the time points, compared to BALB/c animals. A greater bacterial burden was also associated with larger cell counts in the draining lymph node. Other studies using S. epidermidis foreign-body infection models show similar results, with BALB/c mice exhibiting larger CFU counts and/or bigger abscesses, compared to C57BL/6 equivalents (Broekhuizen et al., 2007; Sander et al., 2012). Nevertheless, the number of studies is limited and the topic remains controversial when considering studies using $S$. aureus, which show contradictory results (Chan et al., 2016; Nippe et al., 2011; Nishitani et al., 2015; Prabhakara et al., 2011; von Kockritz-Blickwede et al., 2008). It is known that the immune system of C57BL/6 and BALB/c mice differ from each other (Sellers et al., 2012), with C57BL/6 mice being more Th1 polarised and BALB/C mice being more Th2 polarised. Overall, the current study supported the notion that host genetic background might influence study outcomes and needs to be considered together with the infecting pathogen. Further analysis into immune system differences between these murine strains could provide valuable data regarding the effective immune responses required to clear S. epidermidis infections. Deeper analysis into inflammatory markers at gene and protein level is the subject of ongoing studies in the authors' laboratory, with the aim of characterising the cell environment in the different mechanical contexts. A better understanding of conditions benefiting infection resolution could be of interest in order to improve treatments.

\section{Conclusions}

An S. epidermidis model of FRI was established, which recapitulated many of the clinical features of this infecting agent, such as mild clinical signs and low levels of tissue inflammation. The mice with $S$. epidermidis infection contrasted with animals with $S$. aureus infection, in terms of weight loss, macroscopic observations and cell counts. This model was used to study the impact of fracture stability on infection risk, with stable fixation resulting in a lower infection rate for S. epidermidis in C57BL/6 mice, but not BALB/c mice. In the case of $S$. aureus infection, the rapid osteolysis rendered all fractures unstable and, so, no differences were observed. A more in-depth investigation of these findings is the subject of ongoing studies.

\section{Acknowledgements}

The project was funded by AO Trauma as part of the Clinical Priority Program Bone Infection (grant AR2011_08). The authors would like to acknowledge Tanja Schmid and Iska Dresing for performance of animal surgery, Iris Keller and Pamela Furlong for the bacteriological work and SEM and Nora Goudsouzian and Mauro Bluvol for support with histological processing.

\section{References}

Arens D, Wilke M, Calabro L, Hackl S, Zeiter S, Zderic I, Richards RG, Moriarty TF (2015) A rabbit humerus model of plating and nailing osteosynthesis with and without Staphylococcus aureus osteomyelitis. Eur Cell Mater 30: 148-161.

Augat P, Simon U, Liedert A, Claes L (2005) Mechanics and mechano-biology of fracture healing in normal and osteoporotic bone. Osteoporos Int 16 Suppl 2: S36-43.

Boelens JJ, van der Poll T, Dankert J, Zaat SA (2000) Interferon-gamma protects against biomaterialassociated Staphylococcus epidermidis infection in mice. J Infect Dis 181: 1167-1171.

Broekhuizen CA, de Boer L, Schipper K, Jones CD, Quadir S, Feldman RG, Dankert J, VandenbrouckeGrauls CM, Weening JJ, Zaat SA (2007) Peri-implant tissue is an important niche for Staphylococcus epidermidis in experimental biomaterial-associated infection in mice. Infect Immun 75: 1129-1136.

Campoccia D, Montanaro L, Moriarty TF, Richards RG, Ravaioli S, Arciola CR (2008) The selection of appropriate bacterial strains in preclinical evaluation of infection-resistant biomaterials. Int J Artif Organs 31: 841-847.

Chan LC, Chaili S, Filler SG, Miller LS, Solis NV, Wang H, Johnson CW, Lee HK, Diaz LF, Yeaman MR (2016) Innate immune memory contributes to host defense against recurrent skin and skin structure infections caused by methicillin-resistant Staphylococcus aureus. Infect Immun 26: e00876-16.

Claes L, Recknagel S, Ignatius A (2012) Fracture healing under healthy and inflammatory conditions. Nat Rev Rheumatol 8: 133-143.

Elek SD, Conen PE (1957) The virulence of Staphylococcus pyogenes for man; a study of the problems of wound infection. Br J Exp Pathol 38: 573-586.

Forsberg JA, Potter BK, Cierny G, 3rd, Webb L (2011) Diagnosis and management of chronic infection. J Am Acad Orthop Surg 19 Suppl 1: S8-S19.

Friedrich B, Klaue P (1977) Mechanical stability and post-traumatic osteitis: an experimental evaluation of the relation between infection of bone and internal fixation. Injury 9: 23-29.

Glatt V, Evans CH, Tetsworth K (2016) A Concert between biology and biomechanics: the influence of 
the mechanical environment on bone healing. Front Physiol 7: 678.

Grongroft I, Heil P, Matthys R, Lezuo P, Tami A, Perren S, Montavon P, Ito K (2009) Fixation compliance in a mouse osteotomy model induces two different processes of bone healing but does not lead to delayed union. J Biomech 42: 2089-2096.

Hamers FP, Lankhorst AJ, van Laar TJ, Veldhuis WB, Gispen WH (2001) Automated quantitative gait analysis during overground locomotion in the rat: its application to spinal cord contusion and transection injuries. J Neurotrauma 18: 187-201.

James RC, Macleod CJ (1961) Induction of staphylococcal infections in mice with small inocula introduced on sutures. Br J Exp Pathol 42: 266-277.

Jeffcoach DR, Sams VG, Lawson CM, Enderson BL, Smith ST, Kline H, Barlow PB, Wylie DR, Krumenacker LA, McMillen JC, Pyda J, Daley BJ (2014) Nonsteroidal anti-inflammatory drugs' impact on nonunion and infection rates in long-bone fractures. J Trauma Acute Care Surg 76: 779-783.

Lacroix D, Prendergast PJ (2002) A mechanoregulation model for tissue differentiation during fracture healing: analysis of gap size and loading. J Biomech 35: 1163-1171.

Lankinen P, Lehtimaki K, Hakanen AJ, Roivainen A, Aro HT (2012) A comparative 18F-FDG PET/ CT imaging of experimental Staphylococcus aureus osteomyelitis and Staphylococcus epidermidis foreignbody-associated infection in the rabbit tibia. EJNMMI Res 2: 41.

Laure B, Besnier JM, Bergemer-Fouquet AM, Marquet-Van Der Mee N, Damie F, Quentin R, Favard L, Rosset P (2008) Effect of hydroxyapatite coating and polymethylmethacrylate on stainless steel implant-site infection with Staphylococcus epidermidis in a sheep model. J Biomed Mater Res A 84: 92-98.

Lindsey BA, Clovis NB, Smith ES, Salihu S, Hubbard DF (2010) An animal model for open femur fracture and osteomyelitis: part I. J Orthop Res 28: 38-42.

Lovati AB, Bottagisio M, de Vecchi E, Gallazzi E, Drago L (2017) Animal models of implant-related low-grade infections. A twenty-year review. Adv Exp Med Biol 971: 29-50.

Lovati AB, Drago L, Bottagisio M, Bongio M, Ferrario M, Perego S, Sansoni V, De Vecchi E, Romano CL (2016a) Systemic and local administration of antimicrobial and cell therapies to prevent methicillinresistant Staphylococcus epidermidis-induced femoral nonunions in a rat model. Mediators Inflamm 2016: 9595706.

Lovati AB, Romano CL, Bottagisio M, Monti L, De Vecchi E, Previdi S, Accetta R, Drago L (2016b) Modeling Staphylococcus epidermidis-induced nonunions: subclinical and clinical evidence in rats. PLoS One 11: e0147447.

Magrys A, Paluch-Oles J, Bogut A, Kielbus M, Plewik D, Koziol-Montewka M (2015) The role of programmed death ligand 1 pathway in persistent biomaterial-associated infections. J Microbiol 53: 544-552.

Marmor S, Kerroumi Y (2016) Patient-specific risk factors for infection in arthroplasty procedure. Orthop Traumatol Surg Res 102: S113-119.

Matthys R, Perren SM (2009) Internal fixator for use in the mouse. Injury 40 Suppl 4: S103-109.

Merritt K, Dowd JD (1987) Role of internal fixation in infection of open fractures: studies with Staphylococcus aureus and Proteus mirabilis. J Orthop Res 5: 23-28.

Metsemakers WJ, Kuehl R, Moriarty TF, Richards RG, Verhofstad MH, Borens O, Kates S, Morgenstern $M$ (2016) Infection after fracture fixation: current surgical and microbiological concepts. Injury DOI: 10.1016/j.injury.2016.09.019.

Montanaro L, Speziale P, Campoccia D, Ravaioli S, Cangini I, Pietrocola G, Giannini S, Arciola CR (2011) Scenery of Staphylococcus implant infections in orthopedics. FutureMicrobiol 6: 1329-1349.

Montjovent MO, Siegrist M, Klenke F, Wetterwald A, Dolder S, Hofstetter W (2013) Expression of antagonists of WNT and BMP signaling after nonrigid fixation of osteotomies. Bone 53: 79-86.

Moriarty TF, Debefve L, Boure L, Campoccia D, Schlegel U, Richards RG (2009) Influence of material and microtopography on the development of local infection in vivo: experimental investigation in rabbits. Int J Artif Organs 32: 663-670.

Moucha CS, Clyburn T, Evans RP, Prokuski L (2011) Modifiable risk factors for surgical site infection. J Bone Joint Surg Am 93: 398-404.

Mouzopoulos G, Kanakaris NK, Kontakis G, Obakponovwe O, Townsend R, Giannoudis PV (2011) Management of bone infections in adults: the surgeon's and microbiologist's perspectives. Injury 42 Suppl 5: S18-23.

Nippe N, Varga G, Holzinger D, Loffler B, Medina E, Becker K, Roth J, Ehrchen JM, Sunderkotter C (2011) Subcutaneous infection with S. aureus in mice reveals association of resistance with influx of neutrophils and Th2 response. J Invest Dermatol 131: 125-132.

Nishitani K, Sutipornpalangkul W, de Mesy Bentley KL, Varrone JJ, Bello-Irizarry SN, Ito H, Matsuda S, Kates SL, Daiss JL, Schwarz EM (2015) Quantifying the natural history of biofilm formation in vivo during the establishment of chronic implantassociated Staphylococcus aureus osteomyelitis in mice to identify critical pathogen and host factors. J Orthop Res 33: 1311-1319.

Prabhakara R, Harro JM, Leid JG, Keegan AD, Prior ML, Shirtliff ME (2011) Suppression of the inflammatory immune response prevents the development of chronic biofilm infection due to methicillin-resistant Staphylococcus aureus. Infect Immun 79: 5010-5018.

Riool M, de Boer L, Jaspers V, van der Loos CM, van Wamel WJ, Wu G, Kwakman PH, Zaat SA (2014) Staphylococcus epidermidis originating from 
titanium implants infects surrounding tissue and immune cells. Acta Biomater 10: 5202-5212.

Rittman WW, Perren S (1974) Cortical bone healing after internal fixation and infection. Springer-Verlag Berlin Heidelberg. DOI 10.1007/978-3-642-65977-5.

Robinson DA, Bechtold JE, Carlson CS, Evans RB, Conzemius MG (2011) Development of a fracture osteomyelitis model in the rat femur. J Orthop Res 29: 131-137.

Rochford ET, Sabate Bresco M, Zeiter S, Kluge K, Poulsson A, Ziegler M, Richards RG, O'Mahony L, Moriarty TF (2016) Monitoring immune responses in a mouse model of fracture fixation with and without Staphylococcus aureus osteomyelitis. Bone 83: 82-92.

Sander G, Borner T, Kriegeskorte A, von Eiff C, Becker K, Mahabir E (2012) Catheter colonization and abscess formation due to Staphylococcus epidermidis with normal and small-colony-variant phenotype is mouse strain dependent. PLoS One 7: e36602.

Schafer P, Fink B, Sandow D, Margull A, Berger I, Frommelt L (2008) Prolonged bacterial culture to identify late periprosthetic joint infection: a promising strategy. Clin Infect Dis 47: 1403-1409.

Sellers RS, Clifford CB, Treuting PM, Brayton C (2012) Immunological variation between inbred laboratory mouse strains: points to consider in phenotyping genetically immunomodified mice. Vet Pathol 49: 32-43.

Stadelmann VA, Potapova I, Camenisch K, Nehrbass D, Richards RG, Moriarty TF (2015) In vivo microCT monitoring of osteomyelitis in a rat model. Biomed Res Int 2015: 587857.

Steck R, Ueno M, Gregory L, Rijken N, Wullschleger ME, Itoman M, Schuetz MA (2011) Influence of internal fixator flexibility on murine fracture healing as characterized by mechanical testing and microCT imaging. J Orthop Res 29: 1245-1250.

Stepanovic S, Vukovic D, Dakic I, Savic B, SvabicVlahovic M (2000) A modified microtiter-plate test for quantification of staphylococcal biofilm formation. J Microbiol Methods 40: 175-179.

Trampuz A, Zimmerli W (2006) Diagnosis and treatment of infections associated with fracturefixation devices. Injury 37 Suppl 2: S59-66.

Uckay I, Hoffmeyer P, Lew D, Pittet D (2013) Prevention of surgical site infections in orthopaedic surgery and bone trauma: state-of-the-art update. J Hosp Infect 84: 5-12.

Ueno M, Urabe K, Naruse K, Uchida K, Minehara H, Yamamoto T, Steck R, Gregory L, Wullschleger ME, Schuetz MA, Itoman M (2011) Influence of internal fixator stiffness on murine fracture healing: two types of fracture healing lead to two distinct cellular events and FGF-2 expressions. Exp Anim 60: 79-87.

Versalovic J, Koeuth T, Lupski JR(1991)Distribution of repetitive DNA sequences in eubacteria and application to fingerprinting of bacterial genomes. Nucleic Acids Res 19: 6823-6831.

Vidlak D, Kielian T (2016) Infectious dose dictates the host response during Staphylococcus aureus orthopedic-implant biofilm infection. Infect Immun 84: 1957-1965.

von Kockritz-Blickwede M, Rohde M, Oehmcke S, Miller LS, Cheung AL, Herwald H, Foster S, Medina E (2008) Immunological mechanisms underlying the genetic predisposition to severe Staphylococcus aureus infection in the mouse model. Am J Pathol 173: 16571668.

Willey M, Karam M (2016) Impact of infection on fracture fixation. Orthop Clin North Am 47: 357-364.

Windolf CD, Meng W, Logters TT, MacKenzie CR, Windolf J, Flohe S (2013) Implant-associated localized osteitis in murine femur fracture by biofilm forming Staphylococcus aureus: a novel experimental model. J Orthop Res 31: 2013-2020.

Worlock P, Slack R, Harvey L, Mawhinney R (1988) An experimental model of post-traumatic osteomyelitis in rabbits. Br J Exp Pathol 69: 235-244.

Worlock P, Slack R, Harvey L, Mawhinney R (1994) The prevention of infection in open fractures: an experimental study of the effect of fracture stability. Injury 25: 31-38.

\section{Discussion with Reviewers}

Arianna Lovati: What is the rationale related to the choice of these specific mouse strains?

Authors: These inbred strains were selected for several reasons: first of all the implant model is broadly studied in C57BL/6 mice (Grongroft et al., 2009; Matthys and Perren, 2009; Montjovent et al., 2013; Steck et al., 2011; Ueno et al., 2011), so the desire was to be able to compare our outcomes regarding the bone healing with previous published data. C57BL/6 strain is one of the most used mouse strains and it is very well characterised. C57BL/6 is a Th1-skewed strain and a second strain (Th2-skewed) was added to test if our findings would be independent of genetic background and because we believed that it could provide valuable information for future studies. In addition, both strains are a common background for genetically engineered mice, thus offering interesting future perspectives to assess some of the findings.

Arianna Lovati: Why did you not use mice of the same age to standardise their protocols? Do you think that different ages could have an impact on the experimental outcomes?

Authors: We would like to emphasise that rigid and flexible groups compared were always agematched, with a maximum difference of 4-5 weeks between groups. Skeletally mature mice were used, which is by definition from 20 weeks onward. At this age, animals are considered young adults, so we believed that physiological changes that might occur within a small number of weeks were less relevant in comparison to younger animals in a growing/maturing process, with changes occurring more rapidly. Of note, others (Lu et al., 2005; Lu et al., 2008; additional references) show that differences in 
bone healing are observed mainly between juvenile animals (4 weeks) and young adults (6 months) or elderly mice (18 months), while differences between young adults and elderly mice are modest. This also encouraged us to think that between 5 and 7 months (our range) differences would not be significant.

It may be that age played a role in the outcome of bacterial inoculation in this model. It would be interesting for example to compare juvenile against older mice. However, this would be a significant undertaking and outside the immediate focus of our work.

Edward Schwarz: It appears that the scientific basis of stability is merely implant design. However, as the mouse femur is very small, the plate with the gap may still provide similar rigid fixation as the solid plate in this osteotomy model. Thus, the authors should provide ex vivo biomechanical data demonstrating significant differences in fracture stability to substantiate this novel model.

Authors: We can confirm that the mechanism chosen to control stability was by using implants of different design.

The MouseFix ${ }^{\mathrm{TM}}$ implant system was first developed over 10 years ago, with the specific goal of achieving controlled and distinct stability by implant design. The mechanical properties of these implantbone constructs have already been described in both cadaver mice and after observation of long term in vivo experiments. Grongroft et al. (2009) show in ex vivo mechanical testing that in the vertical plane, the flexible plate achieved only $25 \%$ of the stiffness of the rigid plate (rigid plate: $8.770 .2 \mathrm{Nmm} /$ degree; flexible plate: $2.070 .1 \mathrm{Nmm} /$ degree; $p<0.0005$ in an independent $t$-test). Steck et al. (2011) show that by day 14 , bones fixed with the rigid implant reach $59 \%$ of the stiffness of the contralateral femur compared with only $27 \%$ for equivalents fixed with the flexible implant. Steck et al. (2011) also show by micro-computed tomography that the rigid implant leads to the formation of relatively dense woven bone callus, partially closing the osteotomy gap at day 14 , while the flexible implant leads to the formation of a large callus of low mineral density. Therefore, as the reviewer states, the flexible implant will provide some stability; however, it is significantly less than in the rigid implant according to these publications and results in different healing patterns. The histological sections showed in the current study were consistent with the results of Steck et al. (2011).

Edward Schwarz: Two standard experimental strains of mice were used, which are known to have significant skeletal (femur anatomy and bone volume) and immunological (Th1 vs. Th2) differences. As the study does not contain any outcome measures to interpret these critical variables on fracture infection, the significant differences between the strains found in this study cannot be interpreted.
Authors: We agree that a deeper analysis of immune responses and/or skeletal differences is necessary to fully interpret the observations made between the mice strains. Having stated that, we wish to repeat that bacteriological evaluation was the primary outcome measure within the study and that our aim was to determine whether any mechanics-based observation in one mouse strain was maintained in a second mouse strain. Therefore, independent of the underlying mechanisms involved, we felt that performing the study in two mouse strains was a valid and thorough approach with respect to our primary outcome measure.

Regarding the differences in the skeletal system, it was observed that the characteristic healing patterns established over a decade ago for the rigid and flexible groups were maintained in both mouse strains. And so, although other differences may exist with regards to the skeletal system between these two strains, both displayed the characteristic features of healing expected under conditions of contrasting mechanical stability, validating their use in this study.

Regarding the immunological differences between C57BL/6 and BALB/C, the differences between the two strains are well described in the literature and this was one of the main reasons behind our selection of these two strains. C57BL/6 mice were initially selected as the implants have been characterised in this strain and the desire was to be able to compare our outcomes with previous published data. As C57BL/6 mice are known to be a Th1-skewed strain, a second, Th2-skewed strain (BALB/c) was added to estimate the effects of mechanics on bone healing and infection within these two disparate, yet characterised mouse strains. As mentioned, a deeper analysis of immune responses is on-going in our laboratories in order to gain a better understanding of the immunological processes underlying our findings, which will be the subject of an upcoming manuscript.

Sebastian Zaat: Bacterial staining on histological samples was shown for implant and bone (Fig. 12). To complete the observations, was histology also performed on soft tissue? Particularly in case of $S$. aureus, the soft tissue remained highly culturepositive for up to $14 \mathrm{~d}$. It would be interesting to know where these bacteria resided within the tissue, e.g. in extracellular microcolonies or intracellularly. This is important in view of a possible bacteria reservoir formation, potentially causing later infections.

Authors: Soft tissue sections from S. epidermidisinfected mice were prepared. However, no bacterial cells were observed in the sections searched. Soft tissue load was low (as measured by quantitative bacteriology) and, thus, bacteria observation at this location resulted quite difficult with a histological approach.

With respect to $S$. aureus-infected animals, histology was not performed, as all animals were retained for quantitative bacteriology. Nevertheless, 
in previous publications where $S$. aureus is used (Rochford et al., 2016) is observed that bacteria form microcolonies in the soft tissue, as well as attach to implant and fragments of dead bone.

Sebastian Zaat: In the discussion, the authors indicated that "ongoing work is attempting to provide some understanding of this phenomenon", referring to the phenomenon of enhanced susceptibility to $S$. epidermidis infection in case of fracture instability. Could the authors indicate which type of studies are being performed to gain a better understanding of the mechanisms involved in this high risk infection in case of fracture instability? What is their hypothesis? Authors: Immunological markers are being assessed in order to characterise the local environment. Cytokine and chemokine locally secretion, as well as gene expression analysis at different time points, are being studied. The hypothesis is that differences at a molecular/cellular level can contribute to a higher susceptibility. At the same time, the study aims to assess immune responses towards $S$. epidermidis, as they are poorly described in literature.

\section{Additional references}

Lu C, Hansen E, Sapozhnikova A, Hu D, Miclau T, Marcucio RS (2008) Effect of age on vascularization during fracture repair. J Orthop Res 26: 1384-1389.

Lu C, Miclau T, Hu D, Hansen E, Tsui K, Puttlitz C, Marcucio RS (2005) Cellular basis for age-related changes in fracture repair. J Orthop Res 23: 1300-1307.

Editor's note: The scientific editor for this paper was Juerg Gasser. 